\title{
Efficient and Extensible Quasi-Explicit Modular Nonlinear Multiscale Battery Model: GH-MSMD
}

\author{
Gi-Heon Kim, Kandler Smith,", Jake Lawrence-Simon, and Chuanbo Yang* \\ National Renewable Energy Laboratory, Golden, Colorado 80401, USA
}

\begin{abstract}
Complex physics and long computation time hinder the adoption of computer aided engineering models in the design of largeformat battery cells and systems. A modular, efficient battery simulation model—the multiscale multidomain (MSMD) model—-was previously introduced to aid the scale-up of Li-ion material \& electrode designs to complete cell and pack designs, capturing electrochemical interplay with 3-D electronic current pathways and thermal response. This paper enhances the computational efficiency of the MSMD model using a separation of time-scales principle to decompose model field variables. The decomposition provides a quasi-explicit linkage between the multiple length-scale domains and thus reduces time-consuming nested iteration when solving model equations across multiple domains. In addition to particle-, electrode- and cell-length scales treated in the previous work, the present formulation extends to bus bar- and multi-cell module-length scales. Example simulations are provided for several variants of $\mathrm{GH}$ electrode-domain models.

(C) The Author(s) 2017. Published by ECS. This is an open access article distributed under the terms of the Creative Commons Attribution Non-Commercial No Derivatives 4.0 License (CC BY-NC-ND, http://creativecommons.org/licenses/by-nc-nd/4.0/), which permits non-commercial reuse, distribution, and reproduction in any medium, provided the original work is not changed in any way and is properly cited. For permission for commercial reuse, please email: oa@electrochem.org. [DOI: 10.1149/2.0571706jes] All rights reserved.

(cc) BY-NC-ND
\end{abstract}

Manuscript submitted September 30, 2016; revised manuscript received February 17, 2017. Published March 24, 2017.

In cutting-edge industries such as automotive and aviation, computer models are valuable tools for reducing the cost of product development, improving manufacturing processes, optimizing designs, and implementing advanced controls. Although the global electricdrive-vehicle market is growing rapidly, the lack of a model that can accurately predict a battery's behavior is recognized as a threat to the automotive industry that has been enhancing its dependence on computer models. In a lithium-ion battery, which is the preeminent candidate powering electric-drive vehicles, physiochemical processes take place in intricate geometries over a wide range of time and length scales. The device response of a battery results from complex nonlinear interplays among material characteristics, design variables, and environmental and operational conditions. The multiscale nonlinear nature of battery physics even more critically affects the device behavior as the size of a battery increases. Without understanding the interplays among the interdisciplinary physicochemical processes occurring across varied scales, it is costly to design long-lasting, highperforming, safe, large batteries.

The U.S. Department of Energy's Computer Aided Engineering for Electric Drive Vehicle Battery (CAEBAT) program has supported development of modeling capabilities to help industries accelerate mass-market adoption of electric-drive vehicles and their batteries. In support of the U.S. Department of Energy, National Renewable Energy Laboratory developed the multiscale multidomain (MSMD) model, overcoming challenges in modeling the highly nonlinear multiscale response of battery systems. ${ }^{1,2}$ The MSMD model introduces separate model domains at particle, electrode, and cell levels, while tightly coupling the physics across the scales. The separation of a model domain and the adoption of local homogeneity assumption are enabled by the intrinsic nature of typical battery systems where substantial time- and length-scale segregation occurs. The MSMD particle-domain models (PDMs) solve collective response of electrically and ionically connected particle-batteries which are collocated in the electrode-domain. The electrode-domain models (EDMs) solve the collective behavior of particle-domain batteries, considering polarization through electrolyte and composite matrices. The celldomain models (CDMs) of the MSMD solve single- or multi-cell battery response by resolving the collective behavior of paired plate electrode-domain batteries, considering polarization caused by nonuniform temperature and electric potential fields across cell volume. The MSMD provides a high degree of flexibility and extensibility

*Electrochemical Society Member

${ }^{\mathrm{z} E}$-mail: kandler.smith@nrel.gov through its modularized hierarchical architecture, enabling integrated investigation on the impacts of complex interactions among material, design, and environmental factors on device behavior. To meet the urgent needs of various engineering applications in the industry, significant computation speed improvement of the high fidelity model is greatly desired. The inevitable nested iteration in the MSMD, ensuring self-consistency at each hierarchical level, costs the model computational speed. On the other hand, traditional multiphysics approaches collapse the scales into a single large differential algebraic equation system and apply sophisticated matrix treatments. The hierarchical nature of battery physics renders this collapsed system impractically large and stiff.

Efforts have been made to reduce computational time for solving the battery model, since Doyle et al. $^{4-6}$ presented a macrohomogeneous electrode model. They greatly simplified the model complexity by considering a composite electrode to be a homogeneous porous medium without regard to details of its mesoscale particulate geometry. The active material was often assumed to be made of spherical particles, with diffusion being the mechanism of transport of the lithium. This assumption has been widely accepted and proven. One-dimensional diffusion systems have analytical series solutions for time-varying flux boundary conditions; therefore, previous studies demonstrated successful simplification of modeling transport in solid electrode particles. Doyle et al. ${ }^{4}$ introduced Duhamel's superposition to solve the solid phase Li diffusion model. Wang and Srinivasan ${ }^{7}$ and Subramanian et al. ${ }^{8}$ used polynomial functions for Li concentration profiles in particles. Guo and White ${ }^{9}$ obtained an approximate solution by evaluating an error term to the truncated analytical series solution. Hu et al. ${ }^{10}$ applied the linear time invariant method to numerically obtain a transfer function. In spite of many successes in simplified solid-phase diffusion models, their impact on promoting the computational speed of battery performance prediction has been limited. A few published research studies have addressed model order reduction across the entire system of equations presented by Doyle et al. ${ }^{4}$ Smith et al. ${ }^{3}$ developed a reduced order model (ROM) using a state-space representation of the Doyle's model. It finds the frequency domain transfer functions of lithium diffusion dynamics, charge transfer kinetics, and charge balance through a composite electrode pair for a perturbed applied current. High-frequency responses are truncated and the model order is further reduced with residue grouping and cost-function minimization processes. Cai and White ${ }^{11}$ used proper orthogonal decomposition to find the basis from the solution sets of a high-order model for typical operational modes and truncated triv$\mathrm{ial}$ responses. Lee et al. ${ }^{12}$ presented a direct-realization algorithm to identify a discrete time ROM for a given set of electrochemical model 
parameters at a given state of charge and temperature. The ROMs are useful in various practical applications repeatedly solving a system of differential equations with invariant parameters. However, as soon as the ROM basis is acquired in a reduced dimension space, they easily lose physical interpretations. This makes it difficult to apply the ROMs to a system in which its characteristics evolve. Therefore, the applicability of the model to battery-design optimization or devicedegeneration study is restricted.

This paper presents a new approach for fast and accurate solution of the MSMD battery model. Governing equations for charge conservation are decomposed into fast " $G \times i$ " and slow " $H$ " time varying terms, being partially linearized to provide a quasi-explicit coupling among the multiple model length scales. Equations are derived at the particle-, electrode-, cell-, and module-length scales. Simulation results are presented comparing the full and GH versions of MSMD EDMs.

In the literature, splitting or decomposition concepts are often used to reduce the computational burden of multiphysics multiscale problems. ${ }^{13,14}$ These methods decompose the model into separate physical problems or time scales ${ }^{15}$ that can be solved independently and then linked. Similar to those methods, the present work decomposes the potential field variable $\phi-$ which exists at all length scales of a battery - into slow and fast time scales. Like other works, this speeds up computation of the current/potential relationship at each individual length scale. But unlike any work that we have found, the decomposition also provides an explicit linkage between the separate length scales, eliminating the need to perform separate iterations at each length scale in a nested manner.

\section{Model Description}

GH-formulation.-A mathematical model provides a relation between input and output of a system in context of its states and parameters. A battery model defining a voltage-current relation is presented in Eq. 1,

$$
\phi=f(i ; \boldsymbol{x}, \boldsymbol{p}),
$$

where $\phi$ and $i$ are voltage and current of a battery with $\boldsymbol{x}$ and $\boldsymbol{p}$ for its states and parameters. $f$ is a nonlinear implicit function and is costly to evaluate. If $f$ can be decomposed into a sum of a fast-varying function $g$ and a slow-varying function $h$, then Eq. 1 becomes Eq. 2,

$$
\phi=g(i ; \boldsymbol{x}, \boldsymbol{p})+h(i ; \boldsymbol{x}, \boldsymbol{p})
$$

where the magnitude of $\frac{d h}{d i}$ is substantially smaller than the magnitude of $\frac{d g}{d i}$ by definition. Note that $g$ and $h$ are not uniquely determined. Assuming the existence of a slow-varying function $G$ such that,

$$
G(i ; \boldsymbol{x}, \boldsymbol{p})=\frac{d g}{d i},
$$

Eq. 2 can be written into

$$
\phi=G(i ; \boldsymbol{x}, \boldsymbol{p}) i+H(i ; \boldsymbol{x}, \boldsymbol{p}),
$$

where both $\frac{d G}{d i}$ and $\frac{d H}{d i}$ are negligible. Since both $G$ and $H$ are slowvarying functions, Eq. 4 provides a quasi-explicit relation between $\phi$ and $i$ for given values of $G$ and $H$ at a certain instance. Variable decomposition based on time-scale separation and following partial linearization converts a nonlinear implicit relation in Eq. 1 into a quasiexplicit relation in Eq. 4. In the course of constructing the GH-MSMD quasi-explicit modular nonlinear multiscale model, these procedures are applied in all hierarchical levels of PDMs, EDMs, and CDMs. Note that the function $G$ has a unit of resistance and the function $H$ has a unit of potential. The proper physical interpretations of the quantities $G$ and $H$ can vary depending on the models of choice. While determination of $G$ requires linearization of the governing equation - sometimes associated with loss of accuracy - the $H$ term, determined as $H=\phi-G i$, can capture the residual nonlinearity of the problem and no accuracy is lost. This is similar to solving a nonlinear function using Newton's method where the partial derivative of the function with respect the solution variable need not be exact. The partial derivative simply needs to iteratively advance the solution in the correct direction. The nonlinear function is reevaluated at each iteration ensuring the nonlinearity is not lost. More discussions and specific assumptions applied in GH-MSMD model formulation are provided below.

Baseline submodels. - The modular architecture of the MSMD facilitates unrestricted choice of submodels at each hierarchical model domain. The GH-MSMD inherits the modularity of the MSMD. In this paper, however, baseline submodels are presented and taken for GH-formulation to demonstrate a GH-MSMD construction. A PDM resolves lithium transport in solid electrode particles, interfacial reaction kinetics, and charge conservation at the interfaces. The discrete diffusion particle model (DDPM) is chosen as a baseline PDM addressing the impacts of particulate morphology, size distribution, surface modification, contact resistances, and mixture composition of active particles. A system of particles is considered electronically continuous, but ionically discrete. An arbitrary number of quantized discrete particles can be given as a user input. Thermodynamic, kinetic, transport, electrical, and geometrical model parameters of each discrete particle can be determined independently. An EDM solves electronic and ionic charge conservation in composite electrodes and electrolyte, respectively, and species conservation in electrolyte. Assuming the existence of a local in-plane ensemble average in a finite volume of cell composite, a one dimensional porous electrode model (1DPE) is chosen for a baseline EDM. A CDM solves for temperature and electronic current in current collectors and other passive pathways across cell dimensions. An orthotropic cell composite model is a baseline CDM. A battery cell composite has intricate stratified structures, and the assembly units of paired electrode layers are stacked or wound to build prismatic or cylindrical cells. Macroscopic designs for electrically and thermally configuring cell components greatly affect the physicochemical processes occurring in a battery. Numerical complexity of a model can be significantly reduced by treating the cell-composite as a homogeneous orthotropic continuum. For example, the single potential-pair continuum (SPPC) model treats the stratified cell composite as a homogeneous continuum with orthotropic transport properties, and it resolves temperature and a pair of current collector phase potentials in the volume of the continuum with distinct in-plane and transverse conductivities for heat diffusion and electrical current conduction. Table I summarizes the governing equations for the baseline models presented.

G and $\boldsymbol{H}$ evaluation.- $-G$ and $H$ evaluation is a bottom-up procedure starting from the lowest hierarchical model, i.e. a PDM. With the linearized Eq. T1.1 (Table I), which is valid for many conventional lithium-ion batteries in moderate cycling conditions where the magnitude of charge transfer current density would not much exceed the exchange current density, Eq. T1.2 becomes

$$
\begin{aligned}
\phi_{p e} & =\left(R_{\eta \xi, i}+R_{f \xi, i}\right) i_{\xi, i}^{\prime \prime}+U_{i}^{s} \\
& =G_{\xi, i} i_{\xi, i}^{\prime \prime}+H_{\xi, i},
\end{aligned}
$$

where

$$
R_{\eta \xi, i}=\frac{R T}{F\left(\alpha_{a, i}+\alpha_{c, i}\right) i_{o, i}^{\prime \prime}} .
$$

While defining the output voltage of a particle-domain battery as,

$$
V_{\xi}=\phi_{p e}+R_{f \xi}{ }^{\prime \prime \prime},
$$

substituting Eq. 5 into Eq. T1.5 yields the particle-domain GHequation,

$$
V_{\xi}=G_{\xi} \overline{1}_{\xi}^{\prime \prime}+H_{\xi}
$$

For the DDPM as a PDM, the particle-domain $G_{\xi}$ and $H_{\xi}$ evaluated in Eq. 8 are,

$$
G_{\xi}=\frac{\sum_{i} a_{\xi, i} f_{p, i}}{\sum_{i} \frac{a_{\xi, i} f_{p, i}}{\left(R_{\eta \xi, i}+R_{f \xi, i}\right)}}+R_{f \xi},
$$


Table I. Summary of baseline submodel governing equations chosen in the present study.

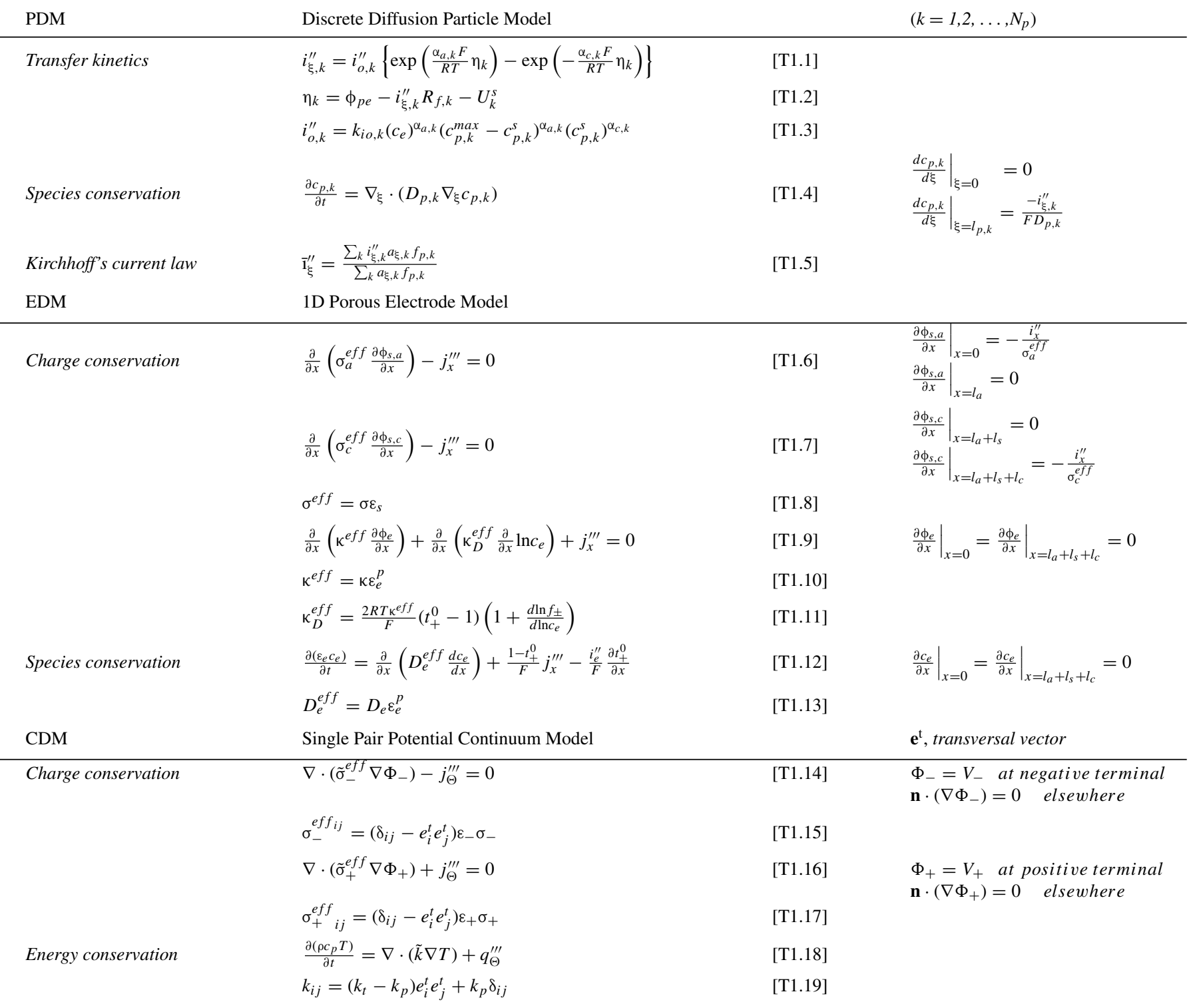

and

$$
H_{\xi}=\frac{\sum_{i} \frac{U_{i}^{s} a_{\xi, i} f_{p, i}}{\left(R_{\eta \xi, i}+R_{f \xi, i}\right)}}{\sum_{i} \frac{a_{\xi, i} f_{p, i}}{\left(R_{\eta \xi, i}+R_{f \xi, i}\right)}} .
$$

Consequently, electrode-domain $G_{x}$ and $H_{x}$ are acquired by reformulating EDM equations for given particle-domain $G_{\xi}$ and $H_{\xi}$. The volumetric current-source profile in the electrolyte phase of electrode composite, $j_{x}^{\prime \prime \prime}$, is evaluated in the EDM with the PDM current density,,$_{\xi}^{\prime \prime}$,

$$
j_{x}^{\prime \prime \prime}=\overline{1}_{\xi}^{\prime \prime} a_{x}
$$

Integrating Eq. T1.6 and Eq. T1.7 over the intervals $\left[0, l_{a}\right]$ and $\left[l_{a}+l_{s}, l_{a}+l_{s}+l_{c}\right]$, respectively, yields the current continuity equations at the anode, Eq. 12, and at the cathode, Eq. 13,

$$
i_{x}^{\prime \prime}=\int_{0}^{l_{a}} j_{x}^{\prime \prime \prime} d x
$$

and

$$
i_{x}^{\prime \prime}=-\int_{l_{a}+l_{s}}^{l_{a}+l_{s}+l_{c}} j_{x}^{\prime \prime \prime} d x
$$

Solid-phase charge-conservation equations Eq. T1.6 and Eq. T1.7 are modified with a homogenized source term and provided in Eq. 14 and Eq. 15.

$$
\begin{gathered}
\frac{\partial}{\partial x}\left(\sigma_{a}^{e f f} \frac{\partial \phi_{s, a}}{\partial x}\right)-\frac{\int_{0}^{l_{a}} j_{x}^{\prime \prime \prime} d x}{l_{a}}=0 \\
\frac{\partial}{\partial x}\left(\sigma_{c}^{e f f} \frac{\partial \phi_{s, c}}{\partial x}\right)-\frac{\int_{l_{a}+l_{s}}^{l_{a}+l_{s}+l_{c}} j_{x}^{\prime \prime \prime} d x}{l_{c}}=0
\end{gathered}
$$

The original model equations simplified the electronic current transport in the composite electrode matrices by introducing porous media properties. The modified equations still give the close solutions of $\phi_{s, a}$ and $\phi_{s, c}$ for typical profiles of $j_{x}^{\prime \prime \prime}$ in conventional systems. Note that Eqs. 14 and 15 also satisfy the current continuity shown in Eqs. 12 and 13. By substituting the solid matrix potential fields $\phi_{s, a}$ and $\phi_{s, c}$ in Eqs. 14 and 15 with their GH-form, such that

$$
\phi_{s, a}=\psi_{s, a} i_{x}^{\prime \prime}+V_{n}, \quad V_{n}=\left.\phi_{s, a}\right|_{x=0}
$$

and

$$
\phi_{s, c}=\psi_{s, c} i_{x}^{\prime \prime}+V_{p}, \quad V_{p}=\left.\phi_{s, c}\right|_{x=l_{a}+l_{s}+l_{c}}
$$


$\psi_{s, a}$ and $\psi_{s, c}$ are solved without depending on $i_{x}^{\prime \prime}$ or boundary values $V_{n}$ and $V_{p}$. If the effective conductivities are uniform at each electrode matrix, then $\psi_{s, a}$ and $\psi_{s, c}$ are given with analytical solutions:

$$
\begin{array}{ccr}
\psi_{s, a}=\frac{l_{a}}{2 \sigma_{a}^{e f f}}\left\{\left(\frac{x}{l_{a}}-1\right)^{2}-1\right\} & \left(0 \leq x \leq l_{a}\right) \\
\psi_{s, c}=\frac{l_{c}}{2 \sigma_{c}^{e f f}}\left\{1-\left(\frac{x-l_{a}-l_{s}}{l_{c}}\right)^{2}\right\} & \left(l_{a}+l_{s} \leq x \leq l_{a}+l_{s}+l_{c}\right)
\end{array}
$$

If effective conductivities are non-uniform, then $\psi_{s, a}$ and $\psi_{s, c}$ are solved with simple numerical calculations with negligible impact to computation speed. Similar modification is done for electrolyte-phase charge-conservation equation Eq. T1.9, as shown in Eq. 20,

$$
\begin{array}{r}
\frac{\partial}{\partial x}\left(\kappa^{e f f} \frac{\partial \phi_{e}}{\partial x}\right)+\frac{\partial}{\partial x}\left(\kappa_{D}^{e f f} \frac{\partial}{\partial x} \ln c_{e}\right)+\frac{\int_{0}^{l_{a}} j_{x}^{\prime \prime \prime} d x}{l_{a}} u(x) u\left(l_{a}-x\right) \\
+\frac{\int_{l_{a}+l_{s}}^{l_{a}+l_{s}+l_{c}} j_{x}^{\prime \prime \prime} d x}{l_{c}} u\left(x-l_{a}-l_{s}\right) u\left(l_{a}+l_{s}+l_{c}-x\right)=0,
\end{array}
$$

where $u(x)$ is a unit step function. By substituting $\phi_{e}$ in Eq. 20 with its $\mathrm{GH}$-form, such that

$$
\phi_{e}=\psi_{e 1} i_{x}^{\prime \prime}+\left(\phi_{e 2}+V_{e L}\right), \quad V_{e L}=\left.\phi_{e}\right|_{x=l_{a}+l_{s}+l_{c}},
$$

we solve for $\psi_{e 1}$ and $\phi_{e 2}$. The analytical solution of $\psi_{e 1}$ for uniform conductivities is shown in Eq. 22,

$$
\psi_{e 1}=\left\{\begin{array}{l}
\left\{1-\left(\frac{x}{l_{a}}\right)^{2}\right\} \frac{l_{a}}{2 \kappa_{a}^{e f f}}+\frac{l_{s}}{\kappa_{s}^{e f f}}+\frac{l_{c}}{2 \kappa_{c}^{e f f}} \quad\left(0 \leq x<l_{a}\right) \\
\left(\frac{l_{a}+l_{s}-x}{l_{s}}\right) \frac{l_{s}}{\kappa_{s}^{e f f}}+\frac{l_{c}}{2 \kappa_{c}^{e f f}} \quad\left(l_{a} \leq x<l_{a}+l_{s}\right) \\
\left(\frac{x-l_{a}-l_{s}-l_{c}}{l_{c}}\right)^{2} \frac{l_{c}}{2 \kappa_{c}^{e f f}} \quad\left(l_{a}+l_{s} \leq x \leq l_{a}+l_{s}+l_{c}\right)
\end{array}\right.
$$

while $\phi_{e 2}$ is given in a simple form with negligible $d \ln f_{ \pm} / d \ln c_{e}$ and $\partial t_{+}^{0} / \partial x$

$$
\phi_{e 2}=\frac{2 R T}{F}\left(1-t_{+}^{0}\right) \ln \frac{c_{e}}{c_{e L}}, \quad c_{e L}=\left.c_{e}\right|_{x=l_{a}+l_{s}+l_{c}} .
$$

Numerical calculations may be used to relax assumptions applied in Eqs. 22 and 23. Substituting the particle-domain GH-equation Eq. 8 into the electrode-domain current continuity equations Eqs. 12 and 13 yields, respectively,

$$
i_{x}^{\prime \prime}=\int_{0}^{l_{a}} a_{x} \frac{V_{\xi}-H_{\xi}}{G_{\xi}} d x
$$

and

$$
i_{x}^{\prime \prime}=-\int_{l_{a}+l_{s}}^{l_{a}+l_{s}+l_{c}} a_{x} \frac{V_{\xi}-H_{\xi}}{G_{\xi}} d x
$$

where the spatial distribution of $V_{\xi}$ is given with,

$$
V_{\xi}=\left\{\begin{array}{ll}
\phi_{s, a}-\phi_{e} & \left(0 \leq x \leq l_{a}\right) \\
\phi_{s, c}-\phi_{e} & \left(l_{a}+l_{s} \leq x \leq l_{a}+l_{s}+l_{c}\right)
\end{array} .\right.
$$

Subsequent substitution of Eqs. 16, 17, and 21 into Eqs. 24 and 25 yields Eqs. 27 and 28.

$$
\begin{gathered}
V_{n}-V_{e L}=\frac{1-\int_{0}^{l_{a}} a_{x} \frac{\psi_{s, a}-\psi_{e 1}}{G_{\xi}} d x}{\int_{0}^{l_{a}} \frac{a_{x}}{G_{\xi}} d x} i_{x}^{\prime \prime}+\frac{\int_{0}^{l_{a}} a_{x} \frac{\phi_{e 2}+H_{\xi}}{G_{\xi}} d x}{\int_{0}^{l_{a}} \frac{a_{x}}{G_{\xi}} d x} \\
=G_{x, a} i_{x}^{\prime \prime}+H_{x, a} \\
V_{p}-V_{e L}=\frac{-1-\int_{l_{a}+l_{s}}^{l_{a}+l_{s}+l_{c}} a_{x} \frac{\psi_{s, c}-\psi_{e 1}}{G_{\xi}} d x}{\int_{l_{a}+l_{s}}^{l_{a}} \frac{a_{x}}{G_{\xi}} d x} i_{x}^{\prime \prime}+\frac{\int_{l_{a}+l_{s}}^{l_{a}+l_{s}+l_{c}} a_{x} \frac{\phi_{e 2}+H_{\xi}}{G_{\xi}} d x}{\int_{l_{a}+l_{s}}^{l_{a}+l_{s}+l_{c}} \frac{a_{x}}{G_{\xi}} d x} \\
=G_{x, c} i_{x}^{\prime \prime}+H_{x, c}
\end{gathered}
$$

The electrode-domain battery working potential $V_{p n}$ is evaluated by subtracting Eq. 27 from Eq. 28.

$$
V_{p n}=V_{p}-V_{n}
$$

Considering plate contact resistance, the electrode-domain battery output voltage is calculated as

$$
\begin{aligned}
V_{x} & =V_{p n}-R_{f x} i_{x}^{\prime \prime} \\
& =\left(G_{x, c}-G_{x, a}-R_{f x}\right) i_{x}^{\prime \prime}+\left(H_{x, c}-H_{x, a}\right) .
\end{aligned}
$$

For the 1-D porous electrode model as EDM, the electrode-domain $\mathrm{GH}-$ equation is,

$$
V_{x}=G_{x} \overline{1}_{x}^{\prime \prime}+H_{x},
$$

where $\overline{1}_{x}^{\prime \prime}=i_{x}^{\prime \prime}$ in the 1-D model and the electrode-domain $G_{x}$ and $H_{x}$ evaluated in Eq. 31 are

$$
G_{x}=-\frac{1+\int_{l_{a}+l_{s}}^{l_{a}+l_{s}+l_{c}} a_{x} \frac{\psi_{s, c}-\psi_{e 1}}{G_{\xi}} d x}{\int_{l_{a}+l_{s}}^{l_{a}} \frac{a_{x}}{G_{\xi}} d x}-\frac{1-\int_{0}^{l_{a}} a_{x} \frac{\psi_{s, a}-\psi_{e 1}}{G_{\xi}} d x}{\int_{0}^{l_{a}} \frac{a_{x}}{G_{\xi}} d x}-R_{f x},
$$

and

$$
H_{x}=\frac{\int_{l_{a}+l_{s}}^{l_{a}+l_{s}+l_{c}} a_{x} \frac{\phi_{e 2}+H_{\xi}}{G_{\xi}} d x}{\int_{l_{a}+l_{s}}^{l_{a}+l_{s}+l_{c}} \frac{a_{x}}{G_{\xi}} d x}-\frac{\int_{0}^{l_{a}} a_{x} \frac{\phi_{e 2}+H_{\xi}}{G_{\xi}} d x}{\int_{0}^{l_{a}} \frac{a_{x}}{G_{\xi}} d x} .
$$

Consequently, cell-domain $G_{\Theta}$ and $H_{\Theta}$ are acquired by reformulating CDM equations for given electrode-domain $G_{x}$ and $H_{x}$. Volumetric current profile in the cell-composite volume, $j_{\Theta}^{\prime \prime \prime}$, is evaluated in the CDM with the EDM plate current density, $\overline{1}_{x}^{\prime \prime}$ :

$$
j_{\Theta}^{\prime \prime \prime}=\overline{1}_{x}^{\prime \prime} a_{\Theta} .
$$

Integrating Eq. T1.14 or Eq. T1.16 over the cell-composite volume yields the current continuity equations at a battery cell,

$$
I_{\Theta}=\int_{\mathcal{V}_{\Theta}} j_{\Theta}^{\prime \prime \prime} d \mathcal{V}
$$

Modified equations of Eqs. T1.14 and T1.16 are given in Eqs. 36 and 37 for charge conservation in negative and positive current collectors:

$$
\begin{aligned}
& \nabla \cdot\left(\tilde{\sigma}_{-}^{e f f} \nabla \Phi_{-}\right)-\frac{\int_{\mathcal{V}_{\Theta}} j_{\Theta}^{\prime \prime \prime} d \mathcal{V}}{\mathcal{V}_{\Theta}}=0 \\
& \nabla \cdot\left(\tilde{\sigma}_{+}^{\text {eff }} \nabla \Phi_{+}\right)+\frac{\int_{\mathcal{V}_{\Theta}} j_{\Theta}^{\prime \prime \prime} d \mathcal{V}}{\mathcal{V}_{\Theta}}=0 .
\end{aligned}
$$

By substituting the current collector potential field $\Phi_{-}$and $\Phi_{+}$in Eqs. 36 and 37 with their GH-form, such that

$$
\Phi_{-}=\Psi_{-} I_{\Theta}+V_{-}, \quad V_{-}=\left.\Phi_{-}\right|_{\text {at negative terminal }}
$$

and

$$
\Phi_{+}=\Psi_{+} I_{\Theta}+V_{+}, \quad V_{+}=\left.\Phi_{+}\right|_{\text {at positive terminal }},
$$

We solve for $\Psi_{-}$and $\Psi_{+}$without dependence on the cell current $I_{\Theta}$ or the boundary values $V_{-}$and $V_{+}$. Substituting the electrode-domain GH-equation Eq. 31 in the cell-domain current-continuity equation Eq. 35 yields,

$$
I_{\Theta}=\int_{\mathcal{V}_{\Theta}} a_{\Theta} \frac{V_{x}-H_{x}}{G_{x}} d \mathcal{V},
$$

where the spatial distribution of $V_{x}$ is given with the relation,

$$
V_{x}=\Phi_{+}-\Phi_{-} .
$$

Subsequent substitution of Eqs. 38 and 39 into Eq. 40 yields,

$$
\begin{aligned}
V_{+-} & =V_{+}-V_{-} \\
& =\frac{1-\int_{\mathcal{V}_{\Theta}} a_{\Theta} \frac{\Psi_{+}-\Psi_{-}}{G_{x}} d \mathcal{V}}{\int_{\mathcal{V}_{\Theta}} \frac{a_{\Theta}}{G_{x}} d \mathcal{V}} I_{\Theta}+\frac{\int_{\mathcal{V}_{\Theta}} a_{\Theta} \frac{H_{x}}{G_{x}} d \mathcal{V}}{\int_{\mathcal{V}_{\Theta}} \frac{a_{\Theta}}{G_{x}} d \mathcal{V}} .
\end{aligned}
$$


Considering additional contact resistance at the cell terminal, the cell output voltage is calculated with the relation,

$$
V_{\Theta}=V_{+-}-R_{f \Theta} I_{\Theta} .
$$

For the SPPC model as the CDM, the cell-domain GH equation is given here,

$$
V_{\Theta}=G_{\Theta} I_{\Theta}+H_{\Theta},
$$

where the cell-domain $G_{\Theta}$ and $H_{\Theta}$ are

$$
G_{\Theta}=\frac{1-\int_{\mathcal{V}_{\Theta}} a_{\Theta} \frac{\Psi_{+}-\Psi_{-}}{G_{x}} d \mathcal{V}}{\int_{\mathcal{V}_{\Theta}} \frac{a_{\Theta}}{G_{x}} d \mathcal{V}}-R_{f \Theta},
$$

and

$$
H_{\Theta}=\frac{\int_{\mathcal{V}_{\Theta}} a_{\Theta} \frac{H_{x}}{G_{x}} d \mathcal{V}}{\int_{\mathcal{V}_{\Theta}} \frac{a_{\Theta}}{G_{x}} d \mathcal{V}} .
$$

Potential and current evaluation.-With the known $G$ and $H$ values evaluated hierarchically from a bottom-up procedure, potential and current evaluation occurs in a top-down manner starting from the highest hierarchical model. A PDM solves the collective response of particle batteries while resolving the interfacial polarization and the electrode diffusion-limiting polarization. An EDM solves the electrode-domain battery response, a collective behavior of particle-domain batteries, considering the polarization through the electrolyte and the composite electrodes. In a CDM, single- or multicell battery response is solved by resolving the collective behavior of electrode-domain batteries for the polarization caused by non-uniform temperature and electric potential fields along current collectors. As the model scale is extended, more causes of polarization are additionally addressed in the model. Therefore, depending on the cell design and the operational condition, either a PDM, an EDM or a CDM can be the model at the highest hierarchy in a MSMD or GH-MSMD simulation. Since $G$ and $H$ are the slow-varying functions, a general GH-equation at the highest hierarchy (Eqs. 8, 31 or 44) provides a quasi-explicit relation between the battery output voltage and the current,

$$
V_{o}=G I_{o}+H
$$

If either $V_{o}$ or $I_{o}$ is known at a certain instance, the other is given explicitly using Eq. 47 . If the battery output power $P_{o}$ or the load resistance $R_{L}$ is given, the voltage and the current outputs are evaluated from simple derivations of Eq. 47 such as

$$
V_{o}=\frac{H+\sqrt{H^{2}+4 G P_{o}}}{2}
$$

or

$$
I_{o}=\frac{H}{R_{L}-G} .
$$

Using the quasi-explicit relations in Eqs. 47-49, the output potential and the current at the highest hierarchical model (i.e., the CDM-SPPC in this study) are evaluated from an electrical load input given as $V_{o}, I_{o}, P_{o}$, or $R_{L}$. With known $V_{\Theta}$ and $I_{\Theta}$ from Eq. 44, Eq. 43 yields $V_{+-}$. Current-collector potentials at the cell terminals $V_{+}$and $V_{-}$are determined by setting one of them as a cell-domain reference; e.g., $V_{-}=0$. Consequently, the current-collector potential fields in a cell volume $\Phi_{+}$and $\Phi_{-}$are found using Eqs. 38 and 39. Spatial distribution of electrode-domain output potential $V_{x}$ is evaluated over a cell-composite volume using Eq. 41. The distribution of electrode-domain plate current density $\overline{\mathbf{1}}_{x}^{\prime \prime}$ is evaluated over a celldomain with the distributions of $G_{x}, H_{x}$, and $V_{x}$ using Eq. 31. With the known $\overline{1}_{x}^{\prime \prime}$, Eq. 27 and Eq. 28 find the electrode-matrix potentials at the current-collector interfaces, $V_{p}$ and $V_{n}$, by having the electrolyte potential at the positive current collector interface $V_{e L}$ as a reference at the electrode-domain; e.g. $V_{e L}=0$. Consequently, $\phi_{s, a}, \phi_{s, c}$, and $\phi_{e}$ are evaluated using Eqs. 16, 17, and 21. Spatial distribution of electrode-domain output potential $V_{\xi}$ is evaluated across the electrode composites using Eq. 26. The distribution of particle-domain current density $\overline{1}_{\xi}^{\prime \prime}$ is evaluated over an electrode domain with the distributions of $G_{\xi}, H_{\xi}$, and $V_{\xi}$ using Eq. 8. With the known $\overline{1}_{\xi}^{\prime \prime}$, Eq. 7 finds $\phi_{p e}$. Finally, the distribution of the interfacial current density at an individual discrete particle, $i_{\xi, i}^{\prime \prime}$, is evaluated using Eq. 5. Note that through the top-down potential and current evaluation procedure, a cell terminal current given at an instance uniquely and explicitly determines the spatial distribution of current in a cell volume and the currents in the lower domain geometries down to the interfacial currents at each particle for the known quasi-static $G$ and $H$ values.

Heat evaluation.-Like the $G$ and $H$ variables, heat is also evaluated hierarchically from a bottom-up procedure. In particle-domain, the DDPM calculates the heat flux from the $i$-th discrete particle interfacial area in Eq. 50.

$$
\begin{aligned}
q_{\xi, i}^{\prime \prime} & =i_{\xi, i}^{\prime \prime 2}\left(R_{\eta \xi, i}+R_{f \xi, i}\right)+i_{\xi, i}^{\prime \prime}\left(U_{i}^{s}-U_{i}^{m}\right)+i_{\xi, i}^{\prime \prime} T \frac{\partial U}{\partial T} \\
& =i_{\xi, i}^{\prime \prime}\left(\phi_{p e}-U_{i}^{m}\right)+i_{\xi, i}^{\prime \prime} T \frac{\partial U}{\partial T}
\end{aligned}
$$

The surface-average particle-domain heat flux is evaluated using Eq. 51, and is carried to the EDM, the upper hierarchical model:

$$
\bar{q}_{\xi}^{\prime \prime}=\frac{\sum_{i} q_{\xi, i}^{\prime \prime} a_{\xi, i} f_{p, i}}{a_{\xi}}+\overline{1}_{\xi}^{\prime \prime 2} R_{f \xi}
$$

For the 1D porous-electrode model, the particle physics contribution to the electrode-domain heat flux is presented based on electrodeplate area in Eq. 52:

$$
q_{x, \xi}^{\prime \prime}=\int_{0}^{l_{a}} a_{x} \bar{q}_{\xi}^{\prime \prime} d x+\int_{l_{a}+l_{s}}^{l_{a}+l_{s}+l_{c}} a_{x} \bar{q}_{\xi}^{\prime \prime} d x .
$$

The ohmic portion of the electrode-domain heat flux is evaluated with Eq. 53. for Eqs. 18, 19, 22, and 23.

$$
\begin{aligned}
q_{x, \Omega}^{\prime \prime}= & \overline{\mathbf{1}}_{x}^{\prime \prime 2}\left(\frac{l_{a}}{3 \sigma_{a}^{e f f}}+\frac{l_{c}}{3 \sigma_{c}^{e f f}}+\frac{l_{a}}{3 \kappa_{a}^{\text {eff }}}+\frac{l_{s}}{\kappa_{s}^{e f f}}+\frac{l_{c}}{3 \kappa_{c}^{e f f}}\right) \\
& +\overline{\mathbf{1}}_{x}^{\prime \prime} \frac{2 R T}{F}\left(1-t_{+}^{0}\right) \ln \frac{\bar{c}_{e, a}}{\bar{c}_{e, c}}
\end{aligned}
$$

The electrode-domain heat flux is evaluated based on the plate area using Eq. 54, and it is carried to the CDM, the upper hierarchical model.

$$
\bar{q}_{x}^{\prime \prime}=q_{x, \xi}^{\prime \prime}+q_{x, \Omega}^{\prime \prime}+\overline{1}_{x}^{\prime \prime 2} R_{f x}
$$

The plate heat flux evaluated in EDM is converted to the volumetric heat source in the cell-composite using Eq. 55.

$$
q_{\Theta, x}^{\prime \prime \prime}=a_{\Theta} \bar{q}_{x}^{\prime \prime}
$$

Equation 56 evaluates the volumetric joule heat source in the cell composite accounting for losses for carrying electronic current along the current collector phases:

$$
q_{\Theta, x}^{\prime \prime \prime}=I_{\Theta}^{2}\left(\tilde{\sigma}_{-}^{e f f} \nabla \Psi_{-} \cdot \nabla \Psi_{-}+\tilde{\sigma}_{+}^{e f f} \nabla \Psi_{+} \cdot \nabla \Psi_{+}\right) .
$$

Note that the terms in the parenthesis in Eq. 56 are invariants in typical occasions. Finally, the total volumetric heat source for the source term in Eq. T1.18 is given as a sum of the heat of EDM and the joule heat at CDM:

$$
q_{\Theta}^{\prime \prime \prime}=q_{\Theta, x}^{\prime \prime \prime}+q_{\Theta, \Omega}^{\prime \prime \prime}
$$

It is often desired to quantify the heat by the cause of heat generation in a battery. Selective formulae are presented for heat categorization in Table II with the given choice of the sub-models in the present study. 
Table II. Heat categorization by cause (PDM-DDPM, EDM-1DPE, CDM-SPPC).

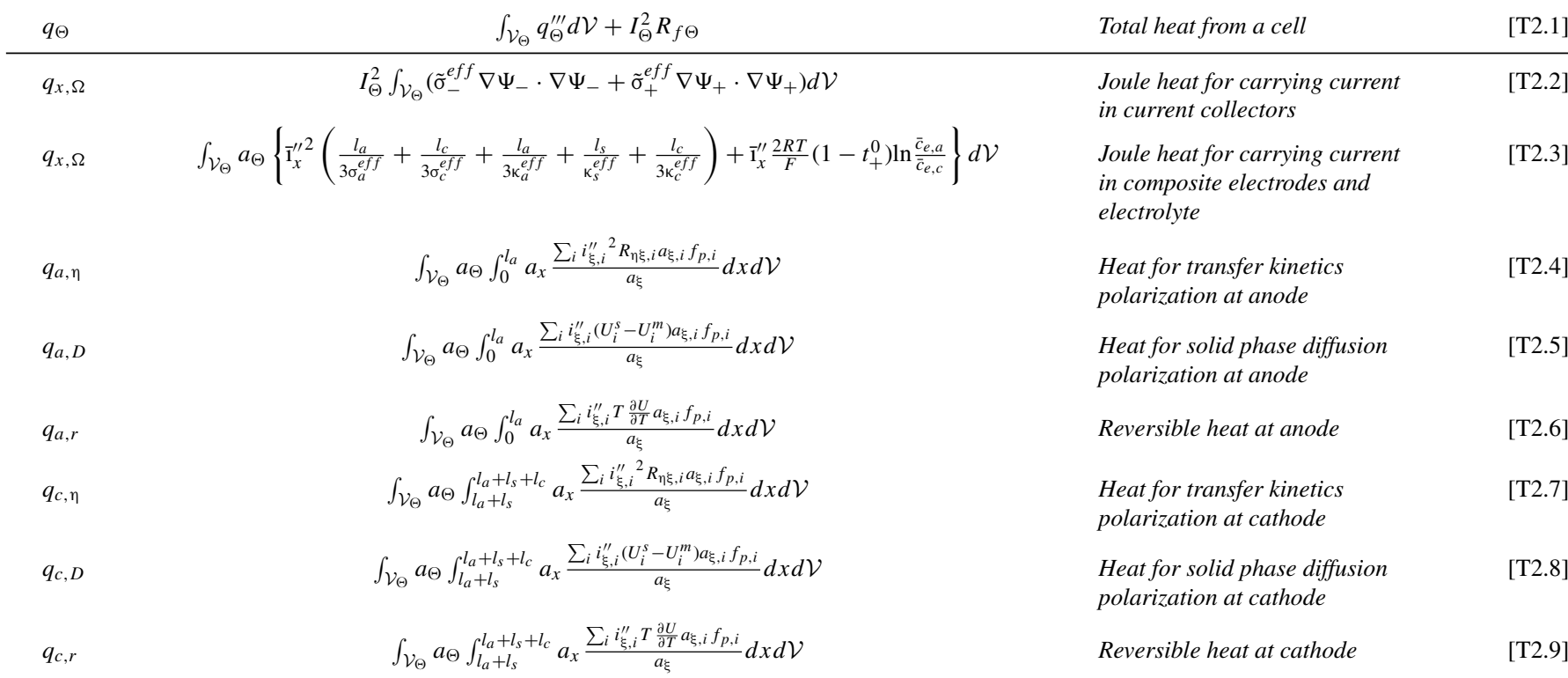

GH-MSMD solution algorithm.-The GH-MSMD inherits the modular architecture of the MSMD, resolving the physicochemical interactions across the scales. Figure 1 compares the modular multi-domain structure of the MSMD and the GH-MSMD. The interdomain coupling between the adjacent length scale submodels in the MSMD occurs by exchanging either domain-invariant or domainaverage quantities without passing geometry-related information. This enables the great modularity and the flexible submodel replacement in the MSMD. However, to ensure self-consistency at each hierarchical level while satisfying current continuity across the domains, multiple layers of nested iteration, which cost the model computational speed, becomes inevitable in the MSMD. In the GH-MSMD, instead of the output current density the slow-varying functions $G$ and $H$ are evaluated in a lower hierarchical model and transferred to a higher hierarchical model. Even though the GH-MSMD results in a very similar multi-domain modular structure as the MSMD, this difference brings critical changes in the model solution algorithm eliminating the need for nested iteration.
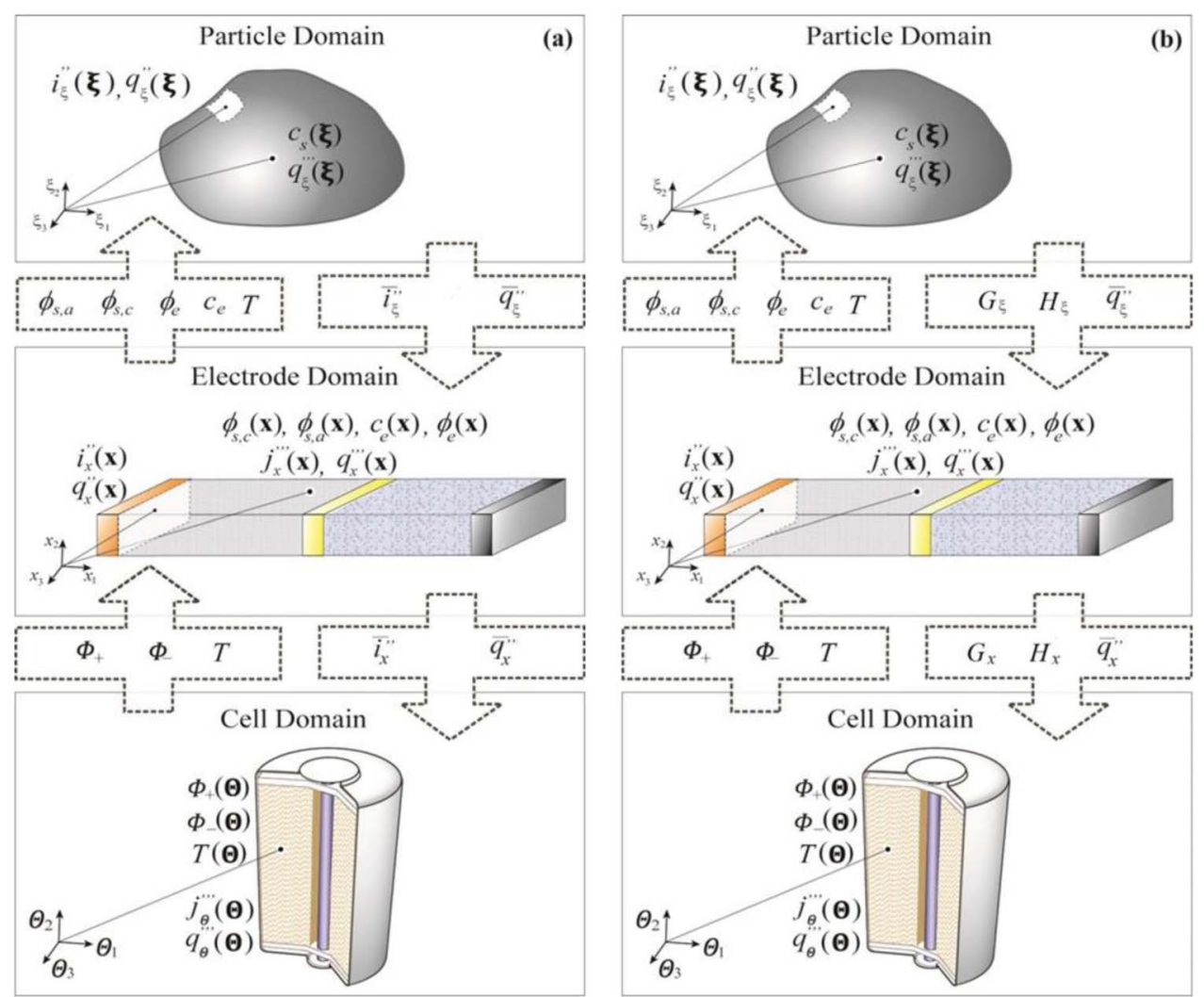

Figure 1. Multi-domain modular structure of the (a) MSMD and (b) GH-MSMD. 


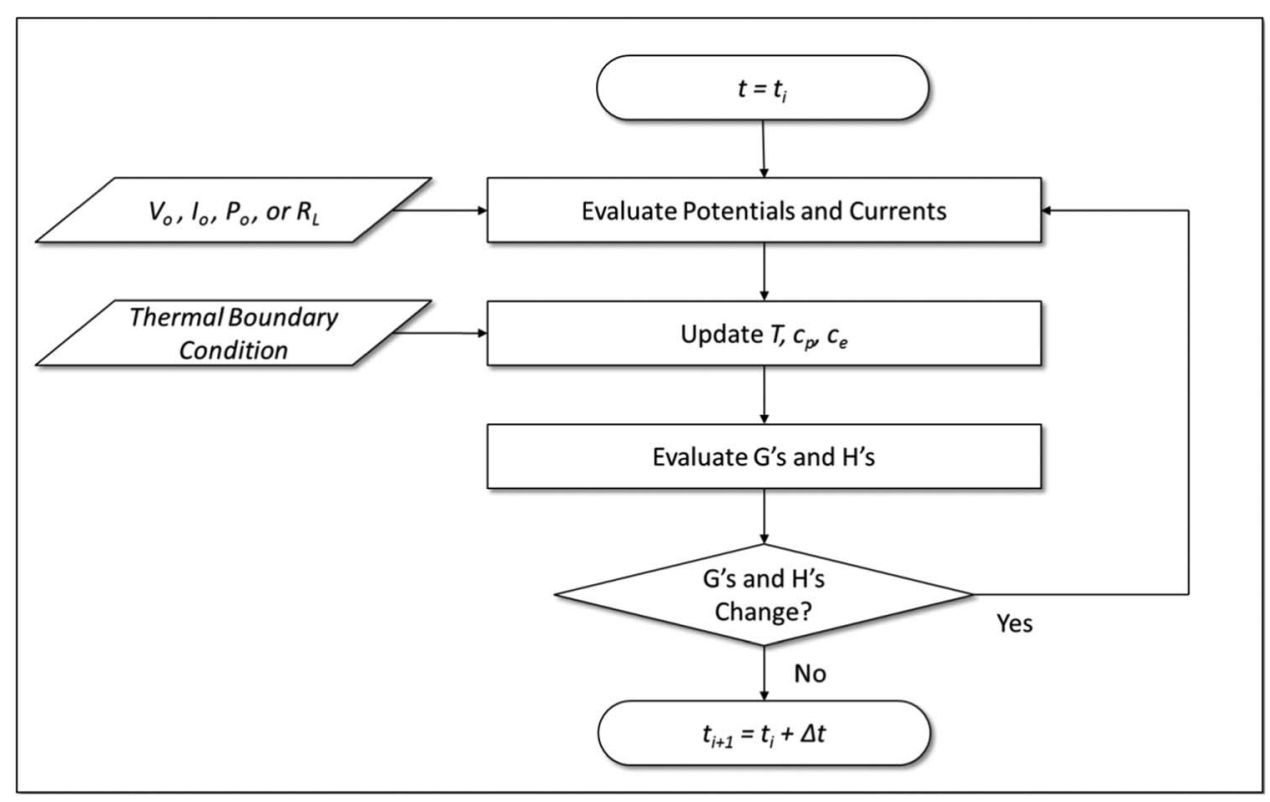

Figure 2. Flowchart for implicit time integration in GH-MSMD.

Figure 2 presents a flowchart for the implicit time integration at $t=t_{i}$ in the GH-MSMD. From the fact that the $G$ and $H$ are slow-varying functions, they (or at least their close approximations) are considered known at any instance. In the beginning of the time integration at $t=t_{i}$, the potential and the current distributions in all domains are explicitly determined for an arbitrarily given electrical load condition with the $G$ and $H$ values known from the previous time-step calculation. In the next step, the battery states-such as temperature and the lithium concentrations in the electrode particles and in the electrolyte - are updated for a given thermal boundary condition and the known potential-current fields. Using the updated battery states, the $G$ and $H$ values are then evaluated explicitly. If the $G$ and $H$ values are meaningfully changed from the previous values, we go back to the first step and iterate the procedures until the $G$ and $H$ values converge. Note that this iteration is not a nested iteration, and it requires only low-cost computation. The converged $G$ and $H$ values are usually acquired after a small number of iteration, because their initial values are already close to the solutions. If the iteration loop is removed from the flowchart shown in Fig. 2, then the potential and current evaluation and the battery-state update are always carried out with the $G$ and $H$ values from the previous time step, which results in the explicit $\mathrm{GH}$ time integration.

Interpretation of $\boldsymbol{G}$ and $\boldsymbol{H}$.-A general GH-equation shown in Eq. 4 is written here without the arguments,

$$
\phi=G i+H .
$$

$G$ is a slow-varying battery state implying the sensitivity of terminal voltage shift for change in external current. $H$ indicates an instantaneous open circuit voltage for deviated equilibriums at local sites. The sign of $G$ is determined depending on whether $i$ is defined as a discharge current or a charge current in the chosen model. In the present study, for example, following the previous practices, the PDM-DDPM defines $\overline{1}_{\xi}^{\prime \prime}$ as a charge current, and the EDM-1DPE and the CDM-SPPC define $\overline{1}_{x}^{\prime \prime}$ and $I_{\Theta}$ as discharge currents. Consequently $G_{\xi}$ is given with positive values, whereas $G_{x}$ and $G_{\Theta}$ have negative values. In Eq. 59, the GH-equation is written for discharge overpotential,

$$
U_{e q}-\phi=-G i+\left(U_{e q}-H\right) .
$$

The first term in the right-hand side of the equation, $-G i$, represents the kinetic and ohmic polarizations. The second term,
$\left(U_{e q}-H\right)$, indicates the polarization due to the deviation of the local thermodynamic equilibria from a system equilibrium, i.e., the polarization for thermodynamic inhomogeneity in a system. $H$ can be defined as a terminal potential at an instance when the terminal current is interrupted. If a system reaches its equilibrium status, $H$ should be equal to $U_{e q}$. $G$ can be defined as a rate of change in potential for change of current. If a system is at its equilibrium, $G$ quantifies a shortpulse resistance. By comparing Eq. 59 with an empirical polarization relation shown in Eq. 60,

$$
U_{e q}-\phi=R_{\text {int }} i
$$

Eq. 61 finds an explicit expression quantifying internal resistance for a non-zero current $i$ :

$$
R_{i n t}=-G+\frac{U_{e q}-H}{i} .
$$

The empirical model Eq. 60 has been widely used in various engineering applications for its simplicity. However, experimental evaluation of $R_{\text {int }}$ in a multi-parameter space requires significant effort, and it results in a model often having large uncertainty. Equation 61 evinces that the GH-MSMD provides a theoretically derived explicit equation for the internal resistance $R_{i n t}$, which has been a conventional empirical quantity. Table III presents the full-blown forms of the cell-domain $G$ and $H$ in Eqs. T3.1 and T3.2 for the given choices of submodels in PDM, EDM, and CDM from Eqs. 9, 10, 32, 33, 45, and 46. These full-blown forms of the $G$ and $H$ function equations do not need to be written in the GH-MSMD, because the choice of the model and evaluation of $G$ and $H$ are completely independent at each domain. Therefore, the inter-domain coupling occurs only by exchanging the evaluated $G$ and $H$. However, the presentation of the full-blown Eqs. T3.1 and T3.2 can provide an insight on how material characteristics, design parameters, and operational and environmental conditions impact the response of a battery, for example, the internal resistance using the explicit equation Eq. 61. This implies that the fully-adaptive, high-fidelity, fast-running, quasi-explicit, modular GH-MSMD would serve as a tool enabling mechanistic investigations for performance evolution of batteries. Equation 62 suggests another interpretation of a $G$ function. If the $\mathrm{GH}$ formulation is carried out in the frequency domain, $\hat{G}$ becomes a transfer function of output potential for a perturbed applied current:

$$
\frac{\Delta \hat{\phi}}{\hat{i}}=\hat{G} .
$$


Table III. Full-blown cell domain $G$ and $H$ with the given submodel choice; PDM-DDPM, EDM-1DPE, and CDM-SPPC.

[T3.1]

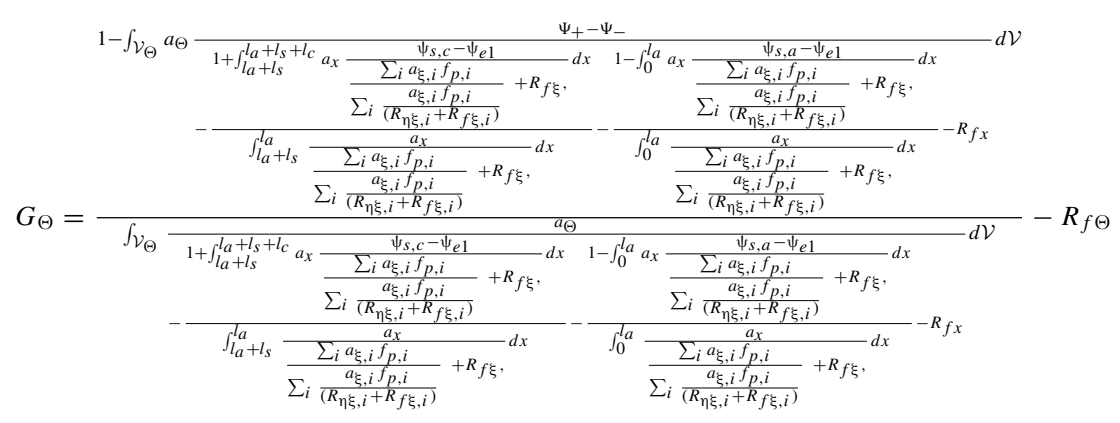

[T3.2]

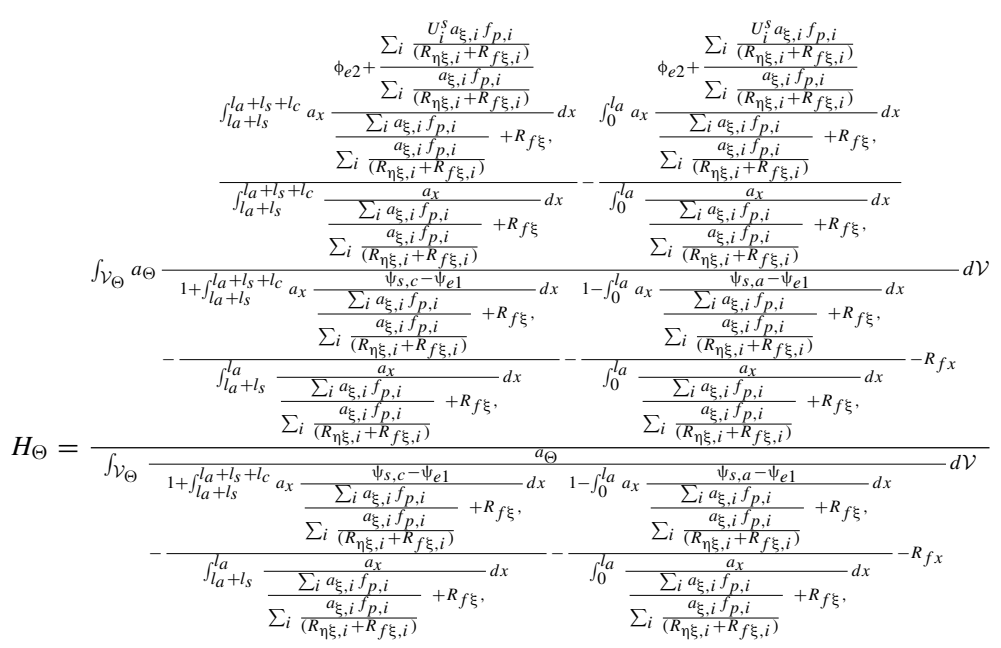

Various derivations of battery states are quantified and used for onboard battery control. It is desired to use physical states for control parameters that are difficult to find from direct measurements. Thanks to greatly enhanced computational speed, the GH-MSMD poses an opportunity to use a physics-based reference model for sophisticated parameter and state estimation in onboard battery management systems. The state of power, for example, is determined in simple practice with the maximum and minimum voltage limits. The maximum discharging or charging power can be evaluated explicitly from the high-fidelity model's $G$ and $H$ using Eqs. 63 and 64:

$$
\begin{array}{r}
\left|P_{o}\right|_{d}^{\max }=\left\{\begin{array}{lr}
\frac{V_{\min }\left(H-V_{\min }\right)}{-G} & (H \leq 2 \\
\frac{H^{2}}{-4 G} & (H>
\end{array}\right. \\
\left|P_{o}\right|_{c}^{\max }=\frac{V_{\max }\left(V_{\max }-H\right)}{-G} .
\end{array}
$$

Simulations.-The accuracy and computational speed of GHMSMD implementations at EDM level are demonstrated by comparing the GH-EDM simulation results with the original MSMD EDM. The original MSMD EDM baseline solves the governing equations T1.1-T1.13 in a traditional segregated solution scheme. Inner iterative loops bringing lower hierarchical domain model to convergence are nested within the next hierarchical outer iteration loops for higher length scale domain model, in turn throughout the multiple layers of the model hierarchy.

Two GH-EDM implementations are presented and verified. The first, denoted as GH-EDM1, solves the baseline submodel governing equations as presented in the previous section; the DDPM for PDM and the 1DPE for EDM. The second, denoted as GH-EDM2, employs the LPD model for EDM replacing the 1DPE with further simplifying assumptions as described in Appendix A. Lumping the particle dynamics while resolving the variation of ohmic polarizations through the electrolyte and the composite electrodes is an effective simplification under dynamic battery operating conditions experienced in applications such as electric vehicles.

Table IV summarizes the EDM parameters used in this study representing a hypothetical 16.5-Ah battery. Additional parameters for thermal simulation are heat transfer surface area $0.2 \mathrm{~m}^{2}$, convection coefficient $10 \mathrm{Wm}^{-2} \mathrm{~K}^{-1}$, and thermal mass $200 \mathrm{~J} \mathrm{~K}^{-1}$. Figure $3 \mathrm{com}-$ pares the original MSMD EDM baseline and the GH-EDMs under constant current discharge simulation at $25^{\circ} \mathrm{C}$. The voltage difference between the original EDM and GH-EDM1 is negligible-generally less than $2 \mathrm{mV}$ - until the end of discharge where kinetic and transport limitations cause a voltage drop off at slightly different capacities as the battery becomes depleted. The GH-EDM2 also retains similar $\sim 2 \mathrm{mV}$ accuracy at C-rates of 5C and below. Figure 4 shows battery internal lithium concentrations in the solid and electrolyte phase for the $5 \mathrm{C}$ discharge simulation. Solid and electrolyte phase concentrations are both well-matched between the baseline and GH-EDM1. The GH-EDM2 model shows minor differences in electrolyte concentration and potential distributions compared to the higher-order models, owing to the lumped treatment of solid state concentrations across the electrode. These differences contribute to a difference in heat generation rate in Figure 3 and thus, slight temperature and end-of-discharge differences for the GH-EDM2 model at the high rate. Current density is not directly calculated in GH-EDM2 and thereby not shown in Figure 4d.

Figure 5 shows constant-current discharge results at $0^{\circ} \mathrm{C}$ with better than 5-mV voltage prediction accuracy. At the 5C rate, the GH-EDM2 
Table IV. Summary of model parameters in the submodel choices.

Particle Domain Parameter

Maximum Li capacity, $c_{p}^{\max }\left[\mathrm{mol} \mathrm{m}^{-3}\right]$

Characteristic diffusion length,.$l_{p}[\mathrm{~m}]$

Stoichiometry at $0 \% \mathrm{SOC}, \theta_{0 \%}$

Stoichiometry at $100 \%$ SOC, $\theta_{100 \%}$

Reference exchange current density at

$100 \%$ SOC, $i_{o, \text { ref }}^{\prime \prime}\left[\mathrm{A} \mathrm{m}^{-2}\right]$

- activation energy, $E_{a c t}^{i_{o}}\left[\mathrm{~J} \mathrm{~mol}^{-1}\right]$

Charge-transfer coefficients, $\alpha_{a}, \alpha_{c}$

Film resistance, $R_{f \xi}\left[\Omega \mathrm{m}^{2}\right]$

Solid diffusion coefficient, $D_{p}\left[\mathrm{~m}^{2} \mathrm{~s}^{-1}\right]$

- activation energy, $E_{\text {act }}^{D_{p}}\left[\mathrm{~J} \mathrm{~mol}^{-1}\right]$

Negative electrode, $U_{a}[\mathrm{~V}]^{*}$

Positive electrode, $U_{c}[\mathrm{~V}]$

Electrode Domain

Parameter

Thickness, $l_{a}, l_{s}, l_{c}[\mathrm{~m}]$

Volume fraction inert, $\varepsilon_{f}$

Volume fraction electrolyte, $\varepsilon_{e}$

Volume fraction active material, $\varepsilon_{s}$

Specific active surface area, $a_{x}\left[\mathrm{~m}^{2} \mathrm{~m}^{-3}\right]$

Solid electronic conductivity, $\sigma_{a}, \sigma_{c}\left[\mathrm{~S} \mathrm{~m}^{-1}\right]$

Bruggeman tortuosity exponent, $p$

Electrolyte concentration, $c_{e}\left[\mathrm{~mol} \mathrm{~m}^{-3}\right]$

Electrolyte $\mathrm{Li}^{+}$diffusion coefficient, $D_{e}\left[\mathrm{~m}^{2} \mathrm{~s}^{-1}\right]$

Electrolyte ionic conductivity, $\kappa\left[\mathrm{S} \mathrm{m}^{-1}\right]$

$\mathrm{Li}^{+}$transference number, $t_{+}^{o}$

Thermodynamic factor, $\partial \ln f_{ \pm} / \partial \ln c_{e}$

Electrode plate area, $\mathrm{m}^{2}$

*All parameters are from Ref. 1 except where noted by asterisk.
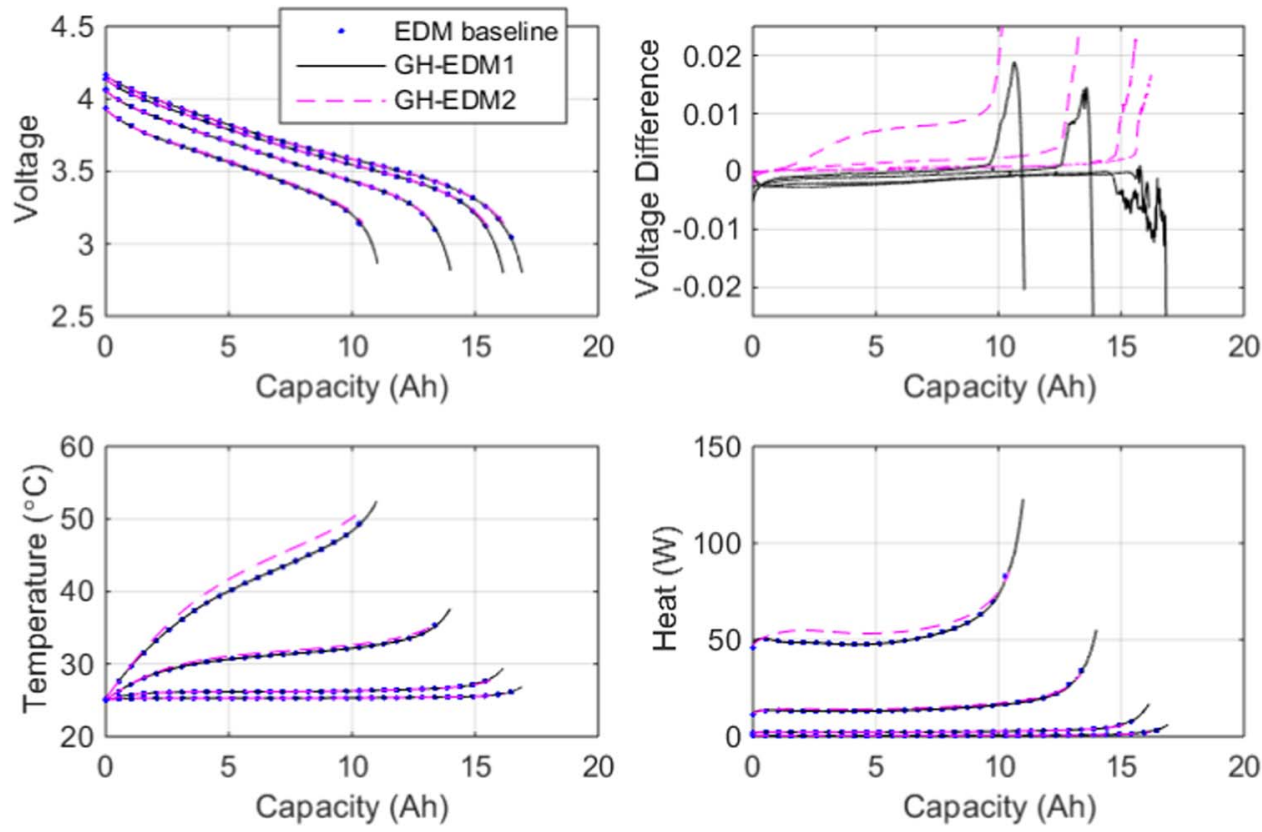

$\left(3.010 \times 10^{6}\right.$

Figure 3. Constant current discharge at $25^{\circ} \mathrm{C}$ and $1 \mathrm{C}, 2 \mathrm{C}, 5 \mathrm{C}, 10 \mathrm{C}$ rates.

Value/Model

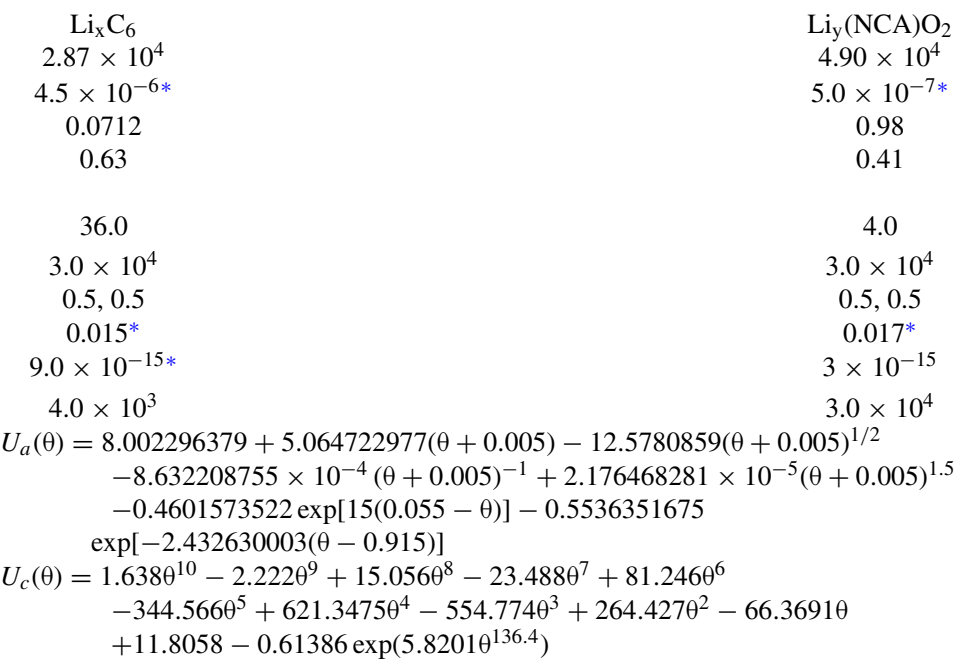

Negative electrode

composite

$70.0 \times 10^{-6}$

Separator

Positive electrode

$0.01^{*}$

0.6 composite

0.40

0.4$$
0.59^{*}
$$

$10.0^{*}$

1.5

$1.2 \times 10^{3}$

$D_{e}=5.84 \times 10^{-7} \exp [-2870 / T]\left(c_{e} / 1000\right)^{2}-33.9 \times 10^{-7} \exp [-2920 / T]\left(c_{e} / 1000\right)$

$+129 \times 10^{-7} \exp [-3200 / T]$

$\kappa=3.45 \exp [-798 / T](c / 1000)^{3}-48.5 \exp [-1080 / T](c / 1000)^{2}$

$+244 \exp [-1440 / T](c / 1000)$

$t_{+}^{0}=-0.000267 \exp [883 / T]\left(c_{e} / 1000\right)^{2}+0.00309 \exp [653 / T]\left(c_{e} / 1000\right)$

$+0.517 \exp [-49.6 / T]$

0

1

0.40

0.41

$\left(0.750 \times 10^{6}\right)$

10

1.5 

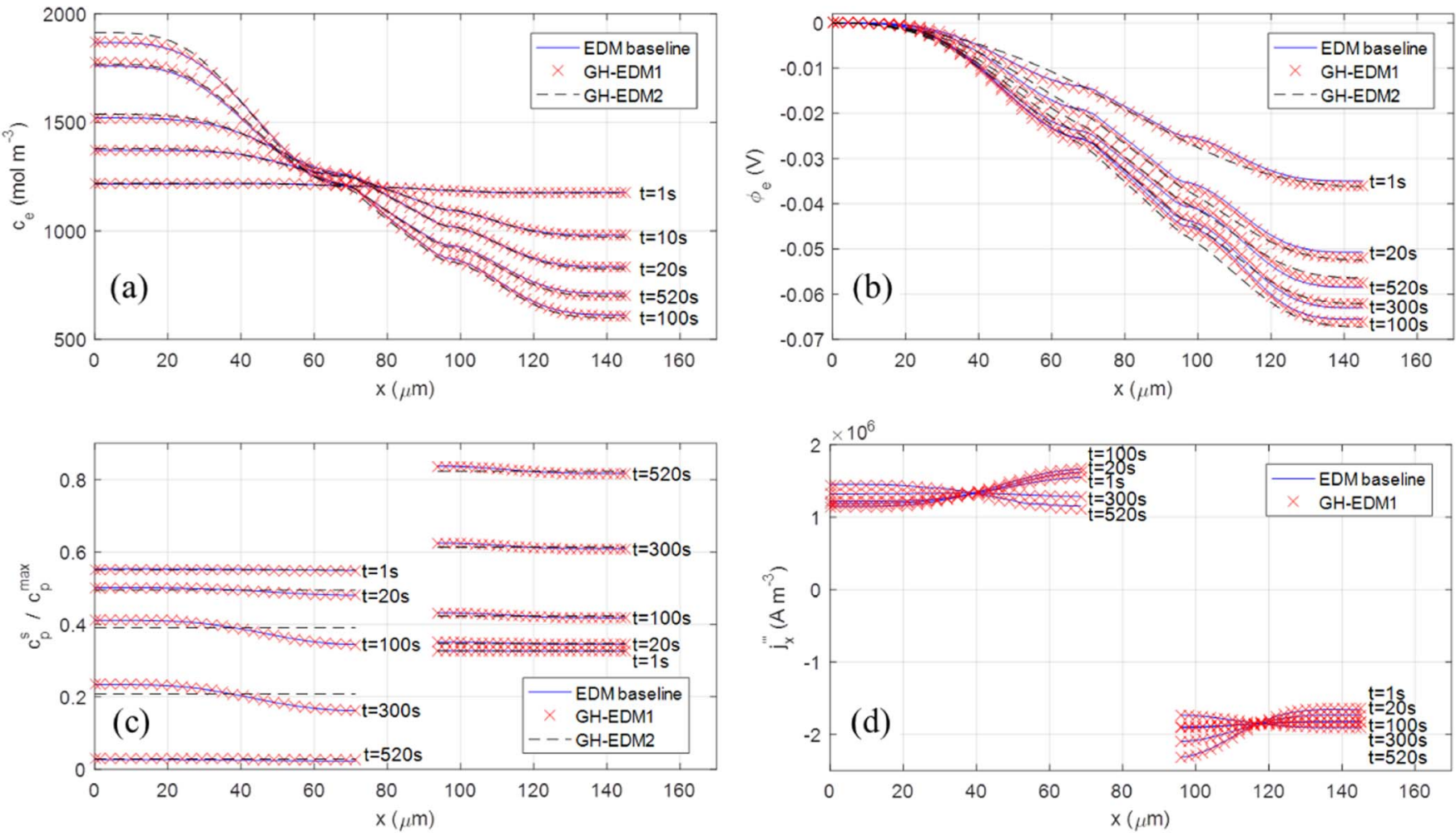

Figure 4. (a) Electrolyte salt concentration, (b) electrolyte phase potential, (c) solid surface concentration, and (d) volumetric current density during $5 \mathrm{C}$ discharge at $25^{\circ} \mathrm{C}$.

model diverges somewhat in heat generation rate and temperature, leading to the voltage difference.

Figure 6 shows simulation results for an electric vehicle drive cycle at $25^{\circ} \mathrm{C}$. The voltage difference between the EDM baseline and $\mathrm{GH}-$ EDM1 models is on the order of $3 \mathrm{mV}$. The GH-EDM2 model agrees within $1 \mathrm{mV}$ of the baseline model. At $0^{\circ} \mathrm{C}$ (Fig. 7), the GH-EDM1 model agreement is on the order of $8 \mathrm{mV}$ whereas the GH-EDM2 model agreement is within $1 \mathrm{mV}$ of the baseline model.

Table V compares the computation time for the three models. All simulations were run on an Apple MacBook Pro laptop computer, with uniform time steps of 1 second to facilitate comparison. Timing results differ slightly with different time-marching schemes. The GH-EDM1
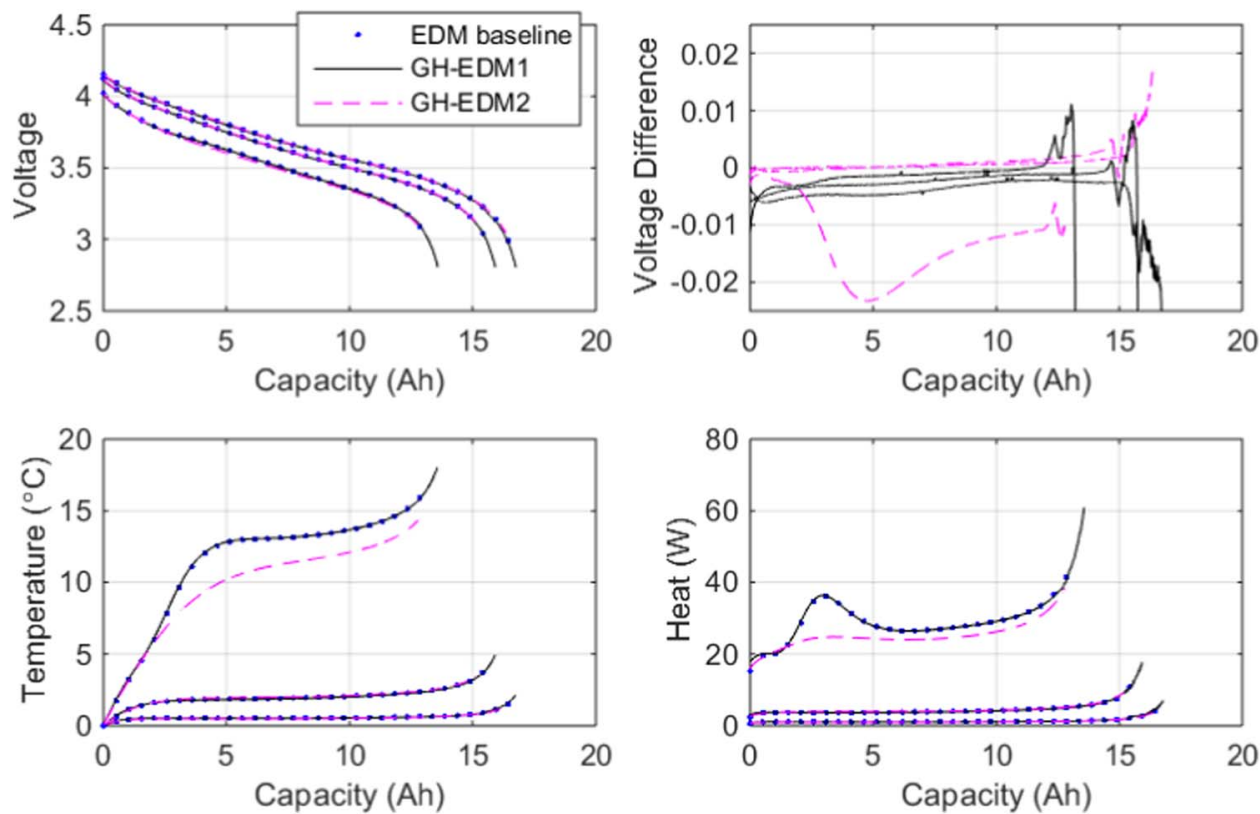

Figure 5. Constant current discharge at $0^{\circ} \mathrm{C}$ and $1 \mathrm{C}, 2 \mathrm{C}, 5 \mathrm{C}$ rates. 

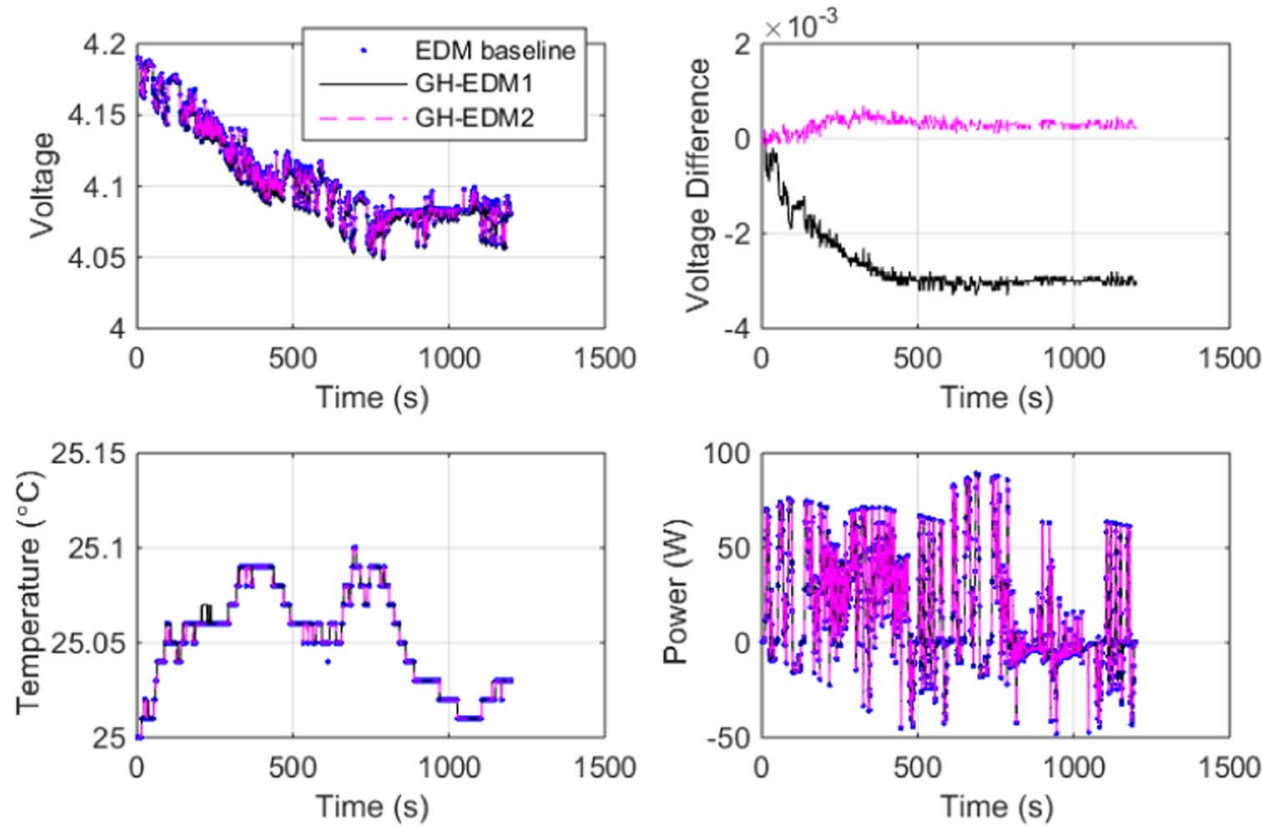

Figure 6. Electric vehicle drive cycle at $25^{\circ} \mathrm{C}$.
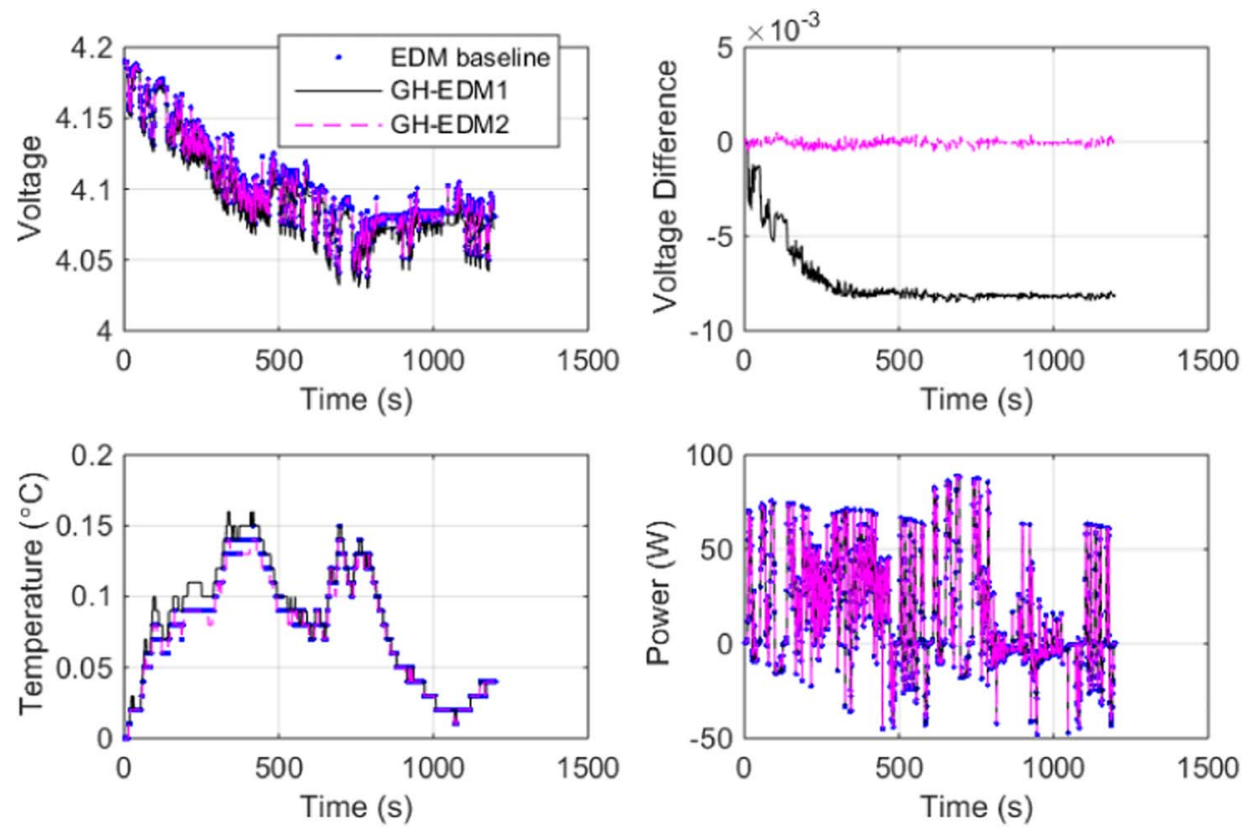

Figure 7. Electric vehicle drive cycle at $0^{\circ} \mathrm{C}$

model is $120-230 x$ faster than the electrode-domain full model. The GH-EDM2 model using the LPD assumption is $800-7000 x$ faster than the full model.

Table V. Computation time for electrode domain full and GH models.

\begin{tabular}{cccccc}
\multicolumn{2}{c}{ Simulation case } & & \multicolumn{3}{c}{ Computation time $(\mathrm{sec})$} \\
\cline { 1 - 2 } \cline { 5 - 6 } $\begin{array}{c}\text { Discharge } \\
\text { profile }\end{array}$ & $\begin{array}{c}\text { Temperature } \\
\left({ }^{\circ} \mathrm{C}\right)\end{array}$ & & $\begin{array}{c}\text { EDM } \\
\text { baseline }\end{array}$ & GH-EDM1 & GH-EDM2 \\
\hline $1 \mathrm{C}$ & 25 & & 360.13 & 3.03 & 0.44 \\
$1 \mathrm{C}$ & 0 & & 816.21 & 3.50 & 0.47 \\
$\begin{array}{c}\text { Drive cycle } \\
\text { Drive cycle }\end{array}$ & 25 & & 1205.92 & 7.06 & 0.83 \\
& 0 & & 8786.45 & 45.00 & 1.27
\end{tabular}

\section{Conclusions}

A new quasi-explicit nonlinear multiscale battery model, GHMSMD, is presented to greatly enhance the computational speed from the previously developed MSMD $^{1}$ model. The MSMD baseline submodel governing equations for 3-D multiphysics simulation of $\mathrm{Li}$-ion battery are reformulated using time scale separation. The decomposition of field variables provides a quasi-explicit linkage between the multiple length scales and eliminates time-consuming nested iteration when solving the MSMD model equations across multiple domains. In addition to particle-, electrode- and cell-length scales, the present formulation is extended to include bus bar and multi-cell module domains (Appendix B). Simulations demonstrate that the GH-MSMD method retains good accuracy while achieving a substantial reduction in computation time. With model computation significantly faster than real time, the GH-MSMD model opens an opportunity to use a 
high fidelity physics model for design optimization, system parameter identification, and real-time control.

\section{Acknowledgments}

This work was supported by the U.S. Department of Energy under Contract No. DE-AC36-08GO28308 with the National Renewable Energy Laboratory. Funding was provided by the U.S. DOE Office of Vehicle Technologies Energy Storage Program, program manager Brian Cunningham. The authors acknowledge Dr. Ahmad Pesaran, the Energy Storage Group manager at NREL for his support. The U.S. Government retains and the publisher, by accepting the article for publication, acknowledges that the U.S. Government retains a nonexclusive, paid-up, irrevocable, worldwide license to publish or reproduce the published form of this work, or allow others to do so, for U.S. Government purposes.

\section{Appendix A: Lumped Particle-Domain (LPD) Model: Reduced-Order EDM}

A PDM captures local thermodynamic deviations caused by particulate inhomogeneity in kinetic, transportive, electrical, geometrical characteristics of electrode material. An EDM captures additional complexity of thermodynamic deviation for mesoscale inhomogeneity caused by electronic and ionic charge transport and mass transport across electrode composite matrices and an electrolyte pore network. The LPD model reduces the order of the 1DPE model by simplifying quantification of polarization for thermodynamic inhomogeneity in a system. The LPD solves the identical EDM equations presented in Table I. By assuming negligible $\partial G_{\xi} / \partial x$ and $\partial H_{\xi} / \partial x$, the integral Eqs. 32 and 33 become the simple algebraic equations,

$$
G_{x}=-\frac{G_{\xi, c}}{a_{x} l_{c}}-\frac{G_{\xi, a}}{a_{x} l_{a}}-\frac{l_{a}}{3 \sigma_{a}^{e f f}}-\frac{l_{c}}{3 \sigma_{c}^{e f f}}-\frac{l_{a}}{3 \kappa_{a}^{e f f}}-\frac{l_{s}}{\kappa_{s}^{e f f}}-\frac{l_{c}}{3 \kappa_{c}^{e f f}}-R_{f x},
$$

and

$$
H_{x}=H_{\xi, c}-H_{\xi, a}+\frac{2 R T}{F}\left(t_{+}^{0}-1\right) \ln \frac{\bar{c}_{e, a}}{\bar{c}_{e, c}} .
$$

This assumption serves well especially for modeling a fairly designed cell used in alternating dynamic-load applications such as electric vehicles, as demonstrated in a previous section. The current continuity Eqs. 12 and 13 become

$$
\overline{\mathbf{1}}_{x}^{\prime \prime}=a_{x} l_{a} \overline{1}_{\xi, a}^{\prime \prime}=-a_{x} l_{c} \overline{1}_{\xi, c}^{\prime \prime},
$$

providing the relation between the electrode-domain plate current density and the particledomain interfacial current density. $V_{\xi}$ and $\phi_{p e}$ are subsequently evaluated using Eq. 8 and Eq. 7. Note that with an assumption for negligible $\left|\nabla G_{x}\right|$ and $\left|\nabla H_{x}\right|$ in the cell domain, a similar computational order reduction method can be applied to a CDM, the Lumped Electrode-Domain (LED) model.

\section{Appendix B: GH-MSMD Extension to a Multi-Cell Battery Model}

Figure B1 suggests a governing equation and its boundary conditions for solving an electrical potential field $\Phi_{b}$ in a three-dimensional passive conductor carrying current $I_{b}$ through the faces $\mathcal{A}_{b 1}$ and $\mathcal{A}_{b 2}$. For a decomposition of $\Phi_{b}$ given in Eq. B1,

$$
\Phi_{b}=I_{b} \Psi_{b}+V_{b 2},
$$

a field $\Psi_{b}$ is acquired independent of $I_{b}$. The solution of $\Psi_{b}$ is then used to evaluate scalar fields $\Phi_{b}$ and $q_{b}^{\prime \prime \prime}$ and a vector field $i_{b}^{\prime \prime}$ without a need for repeatedly solving the Laplace equation for arbitrary transient $I_{b}$ in a conductor geometry. By defining $R_{b}$ as

$$
R_{b}=\int_{\mathcal{V}_{b}} \sigma_{b} \nabla \Psi_{b} \cdot \nabla \Psi_{b} d \mathcal{V}
$$

the total heat generation from a given passive conductor is quantified as,

$$
q_{b}=I_{b}^{2} R_{b} .
$$

The divergence theorem yields the equality between the surface average of $\Psi_{b}$ at the face $\mathcal{A}_{b 1}$ and $R_{b}$,

$$
\bar{\Psi}_{b 1}=\frac{\int_{\mathcal{A}_{b 1}} \Psi_{b} d \mathcal{A}}{\mathcal{A}_{b 1}}=R_{b} .
$$

Consequently the conductor potential drop between the faces $\mathcal{A}_{b 1}$ and $\mathcal{A}_{b 2}$ is evaluated

$$
\bar{V}_{b 1}-V_{b 2}=I_{b} R_{b}
$$

With $R$ values evaluated using Eqs. B2 or B4 for passive conductors and given $G_{\Theta}$ and $H_{\Theta}$ values of individual cells in a multi-cell module, a circuit representation of a $n \mathrm{P} m \mathrm{~S}$ battery module is presented in Fig. B2. The module GH-equation for this configuration is given as

$$
V_{M}=G_{M} I_{M}+H_{M}
$$

where

$$
G_{M}=\sum_{i=1}^{m} \frac{1}{\sum_{j=1}^{n} \frac{1}{G_{\Theta(i, j)}-R_{\Theta(i, j)}}}-\sum_{j=1}^{m-1} R_{b, i}
$$

and

$$
H_{M}=\sum_{i=1}^{m} \frac{\sum_{j=1}^{n} \frac{H_{\Theta(i, j)}}{G_{\Theta(i, j)}-R_{\Theta(i, j)}}}{\sum_{j=1}^{n} \frac{1}{G_{\Theta(i, j)}-R_{\Theta(i, j)}}}
$$

Once $V_{M}$ and $I_{M}$ are evaluated using Eqs. 47-49, the output potential at the $i$-th parallel bank in the module is evaluated as

$$
V_{M, i}=\frac{1}{\sum_{j=1}^{n} \frac{1}{G_{\Theta(i, j)}-R_{\Theta(i, j)}}} I_{M}+\frac{\sum_{j=1}^{n} \frac{H_{\Theta(i, j)}}{G_{\Theta(i, j)}-R_{\Theta(i, j)}}}{\sum_{j=1}^{n} \frac{1}{G_{\Theta(i, j)}-R_{\Theta(i, j)}}} .
$$

Subsequently the individual cell current is identified with

$$
I_{\Theta(i, j)}=\frac{V_{M, i}-H_{\Theta(i, j)}}{G_{\Theta(i, j)}-R_{\Theta(i, j)}} .
$$
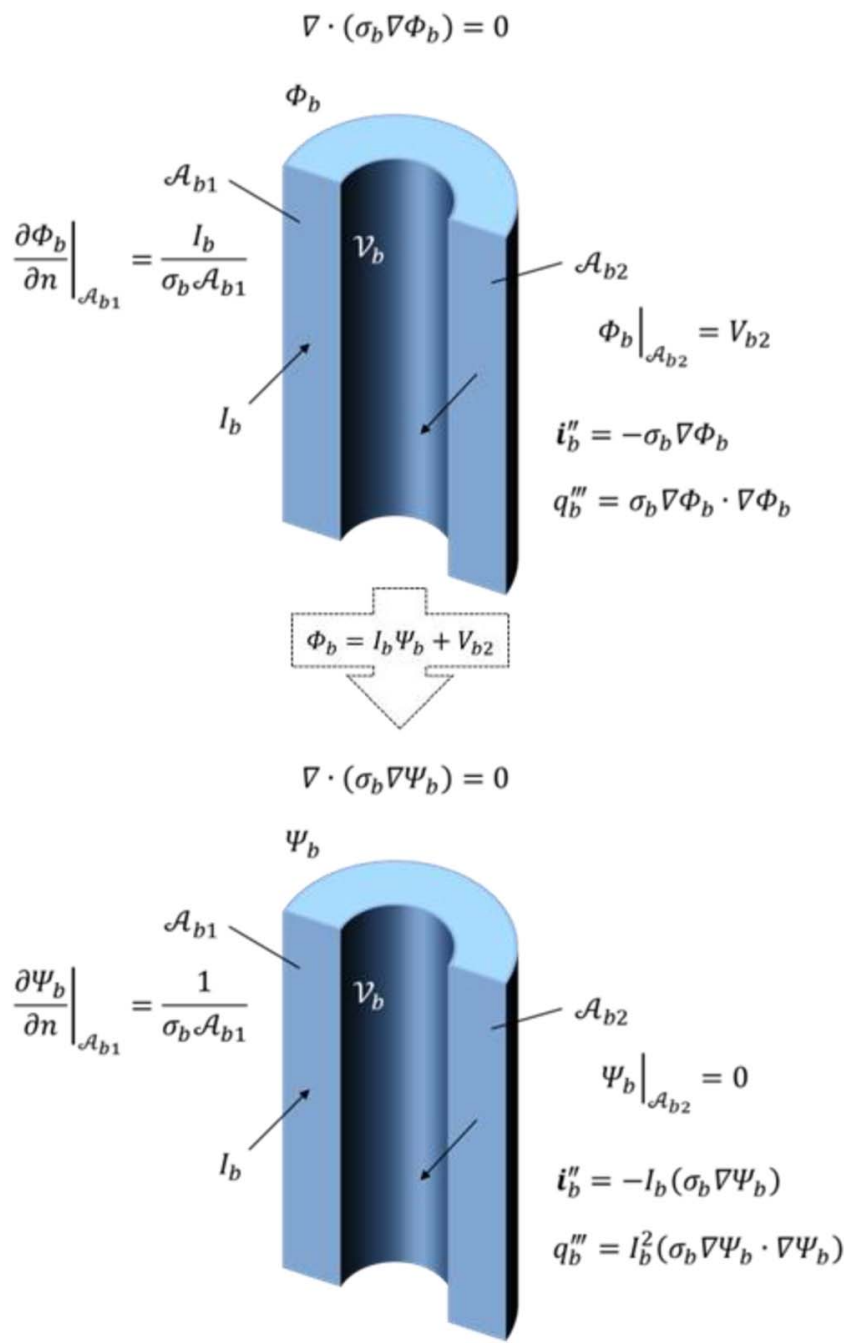

Figure B1. 3-D current carrying conductor. 


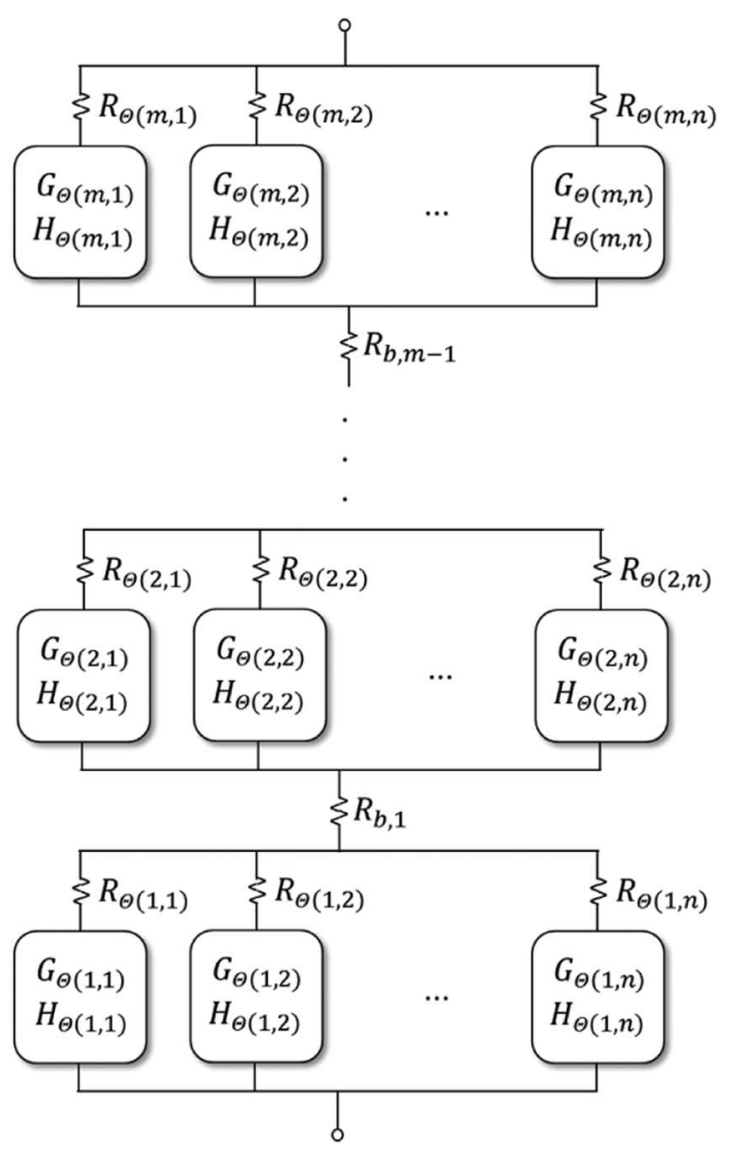

Figure B2. Multi-cell module with cells in $n \mathrm{P} m \mathrm{~S}$ arrangement.

\section{List of Symbols}

Variables

$\begin{array}{ll}a & \text { Volume-specific interfacial area, } \mathrm{m}^{2} \mathrm{~m}^{-3} \\ c & \text { Concentration, mol } \mathrm{m}^{-3} \\ D & \text { Diffusivity, } \mathrm{m}^{2} \mathrm{~s}^{-1} \\ \mathbf{e}^{t} & \text { Unit transverse direction vector } \\ f_{p} & \text { Particle volume fraction } \\ f_{ \pm} & \text {Activity coefficient } \\ F & \text { Faraday constant, } 96485 \mathrm{~A} \mathrm{~s} \mathrm{~mol}^{-1} \\ g & \text { Fast-varying function } \\ G & \text { Fast-varying function, impedance term } \\ h & \text { Slow-varying function } \\ H & \text { Slow-varying function, quasi-static term } \\ I & \text { Current, A } \\ i & \text { Interfacial current density, A m }{ }^{-2} \\ j & \text { Volumetric current density, A m } \\ l & \text { Thickness, } \mathrm{m} \\ \mathbf{n} & \text { Unit normal vector } \\ p & \text { Bruggeman tortuosity exponent } \\ \mathbf{p} & \text { Parameter array } \\ R & \text { Universal gas constant, 8.314 } \mathrm{J} \mathrm{mol}^{-1} \\ R_{i} & \text { Resistance, } \Omega \mathrm{m}^{2}\end{array}$

Time, s

Transference number

Temperature, $\mathrm{K}$

Voltage, $\mathrm{V}$

EDM spatial dimension, $\mathrm{m}$

State array

\section{Greek}

Transfer coefficient

Kronecker delta

Volume fraction

Ionic conductivity, $\mathrm{S} \mathrm{m}^{-1}$

Overpotential, V

Stoichiometry

Potential, V

Electronic conductivity, $\mathrm{S} \mathrm{m}^{-1}$

\section{Subscripts}

Negative electrode/Anode/Anodic

Positive electrode/Cathode/Cathodic

Electrolyte phase

Film

Particle index

Module domain

Reference value

Particle

Solid-phase/separator region

Electrode domain

Cell domain

Particle domain

Negative foil/terminal/bus bar

Positive foil/terminal/bus bar

\section{Superscripts}

eff $\quad$ Effective property

$m \quad$ Mean quantity

$s \quad$ Surface quantity

"I Interfacial quantity

III Volumetric quantity

\section{References}

1. G.-H. Kim, K. Smith, K.-J. Lee, S. Santhanagopalan, and A. Pesaran, J. Electrochem. Soc., 158, A955 (2011).

2. K.-J. Lee, K. Smith, A. Pesaran, and G.-H. Kim, J. Power Sources, 241, 20 (2013).

3. K. A. Smith, C. D. Rahn, and C.-Y. Wang, Energy Convers. Manage., 48, 2565 (2007).

4. M. Doyle, T. Fuller, and J. Newman, J. Electrochem. Soc., 140, 1526 (1993).

5. T. Fuller, M. Doyle, and J. Newman, J. Electrochem. Soc., 141, 1 (1994).

6. M. Doyle and J. Newman, J. Appl. Electrochem., 27, 846 (1997).

7. C.-Y. Wang and V. Srinivasan, J. Power Sources, 110, 364 (2002).

8. V. Subramanian, J. Ritter, and R. White, J. Electrochem. Soc., 148, E444 (2001).

9. M. Guo and R. E. White, J. Power Sources, 198, 322 (2012).

10. X. Hu, S. Stanton, L. Cai, and R. E. White, J. Power Sources, 218, 212 (2012).

11. L. Cai and R. E. White, J. Electrochem. Soc., 156, A154 (2009).

12. J. L. Lee, A. Chemistruck, and G. L. Plett, J. Power Sources, 220, 430 (2012).

13. J. Geiser, J. Algorithms \& Computational Technology, 9, 65 (2015)

14. J. Geiser, Multicomponent and Multiscale Systems: Theory, Methods and Applications in Engineering, Springer, 2016.

15. R.I. McLachlan and G.R.W. Quispel, Acta Numerica, 11, 341 (2002) 